\title{
Coherence-enhanced, phase-dependent dissipation in long SNS Josephson junctions: revealing Andreev Bound States dynamics
}

\author{
B. Dassonneville ${ }^{1}$, A. Murani ${ }^{1}$, M. Ferrier ${ }^{1}$, S. Guéron ${ }^{1}$ and H. Bouchiat ${ }^{1}$ \\ ${ }_{1}^{1}$ LPS, Univ. Paris-Sud, CNRS, UMR 8502, F-91405 Orsay Cedex, France
}

(Dated: May 31, 2021)

\begin{abstract}
One of the best known causes of dissipation in ac driven quantum systems stems from photon absorption causing transitions between levels. Dissipation can also be caused by the retarded response to the time-dependent excitation, and in general gives insight into the system's relaxation times and mechanisms. Here we address the dissipation in a mesoscopic normal wire with superconducting contacts, that sustains a dissipationless supercurrent at zero frequency and that may therefore naively be expected to remain dissipationless at frequency lower than the superconducting gap. We probe the high frequency linear response of such a Normal/Superconductor (NS) ring to a time-dependent flux by coupling it to a highly sensitive multimode microwave resonator. Far from being the simple, dissipationless, derivative of the supercurrent-versus-phase relation, the ring's ac susceptibility also displays a dissipative component whose phase dependence is a signature of the dynamical processes occurring within the Andreev spectrum. We show how dissipation is driven by the competition between two mechanisms. The first is the relaxation of the Andreev levels distribution function, while the second correspond to microwave- induced transitions within the spectrum. Depending on the relative strength of those contributions, dissipation can be maximal at $\pi$, a phase at which the proximity-induced minigap closes, or can be maximal near $\pi / 2$, a phase at which the dc supercurrent is maximal. We also find that the dissipative response paradoxically increases at low temperature and can even exceed the normal state conductance. The results are successfully confronted with theoretical predictions of the Kubo linear response and time-dependent Usadel equations, derived from the Bogoliubov-de Gennes Hamiltonian describing the SNS junction. These experiments thus demonstrate the power of the ac susceptibility measurement of individual hybrid mesoscopic systems in probing in a controlled way the quantum dynamics of Andreev bound states. By spanning different physical regimes, our experiments provide a unique access to inelastic scattering and spectroscopy of an isolated quantum coherent system, and reveal the associated relaxation times. This technique should be a tool of choice to investigate topological superconductivity and detect the topological protection of edge states.
\end{abstract}

PACS numbers:

\section{INTRODUCTION}

Phase coherent rings threaded by an Aharonov-Bohm flux are known to exhibit non-dissipative, persistent currents. This is true of both non-superconducting (normal) mesoscopic rings [1-4] and hybrid Normal metal - Superconductor (NS) rings [5, 6]. In both cases the thermodynamic non-dissipative current results from the phase sensitivity of the system's eigenenergies. The profound analogy between the two systems has been noted since the early predictions of persistent currents in normal rings [7]. It stems from phase-dependent boundary conditions that induce a phase-dependent spectrum. The phase $\varphi$ is linked to the Aharonov-Bohm flux $\Phi$ via $\varphi=-2 \pi \frac{\Phi}{\Phi_{0}}$, where $\Phi_{0}$ is the normal flux quantum $h / e$ in the case of a pure normal ring, and is the superconducting flux quantum $h / 2 e$ in the case of an NS hybrid ring. The current-phase relation at equilibrium has been measured by applying a static flux, corresponding to a dc phase bias $[2,3,6,8,9]$. In contrast, the investigation of the dynamics of these systems is a more recent experimental and theoretical endeavor [10-15]. The tool of choice is the measurement of the magnetic susceptibility $\chi=\delta I_{a c} / \delta \Phi_{a c}$ which relates the current response $\delta I_{a c}$ to a time-dependent ac flux excitation $\delta \Phi_{a c} \exp -i \omega t$.

The onset of dissipation in a phase-driven system, measured by the real part $\mathrm{G}$ of its admittance $Y=\chi / i \omega$, is still an open question. There have been two principal theoretical approaches to the problem of the conductance of a phase coherent sample. The first is the Kubo approach derived from the calculation of the linear response of a conductor to an ac electromagnetic field. In this approach, irreversibility is brought about by coupling the electronic system to a thermal reservoir with a large number of degrees of freedom (e.g. a phonon bath). The second is the Landauer-Büttiker approach in which the conductance of a mesoscopic system connected to electron reservoirs is equal to the transmission coefficient. The equivalence between these two definitions of the conductance has been demonstrated [17] in the case of a voltage-biased system with a continuous spectrum. In that case the conductance is proportional to the elastic scattering time with small quantum corrections related to the phase coherence time. However it has been shown that this equivalence is not valid anymore in a discrete spectrum system [18]. What happens then when an electronic system is isolated from incoherent reservoirs, either with the use of a ring without contacts or via su- 
perconducting contacts ?

Landauer and Büttiker addressed this basic question in a pioneering work $[19,20]$ considering a loop geometry. They predicted that the response to an ac flux should contain a dissipative component of admittance due to the delayed relaxation of populations back to the instantaneous equilibrium value. This flux-dependent ac conductance $G(\Phi)$ is proportional to the inelastic scattering time, contrary to the Drude conductance of a connected system that is related to the elastic scattering time.

This prediction, made in the context of mesoscopic normal metallic rings, could not be observed experimentally even in $10^{5}$ rings $[21,22]$ due to the smallness of the persistent current in normal diffusive mesoscopic rings. In the present paper we focus on the case of a single hybrid NS rings, where this fundamental difference between voltage biasing with reservoirs and flux biasing in a ring geometry persists in the limit of systems with a continuous spectrum. The signature of this phase-dependent dissipation is much greater thanks to the spectral correlations borne from the superconducting boundary conditions. This allows us to reveal the full phase and frequency dependence explored in this article.

In addition to addressing the basic question of the conductance of an isolated electronic system, our work sheds new light on the physics of a large number of Andreev Bound States, microscopic degrees of freedom of Josephson junctions that can be used to perform quantum computation [23-25].

Due to the energy gap $\Delta$ in the superconducting electrodes, a low energy electron (hole) is retro-reflected into a hole (electron) at the interface between the $\mathrm{N}$ and $\mathrm{S}$ metals in a process called Andreev reflection. This process leads to the emergence of Andreev bound states (ABS), superpositions of electron and hole states confined in the $\mathrm{N}$ part of the junction at energies below the superconducting gap. These states were observed by spectroscopy experiments [26-29] in junctions with few channels. They are phase-dependent and carry the Josephson supercurrent $I_{J}(\varphi)=\sum f_{n}(\varphi) i_{n}(\varphi)$ where $f_{n}$ is the Fermi distribution function at energy $\epsilon_{n}(\varphi)$ and the state of energy $\epsilon_{n}$ carries a current $i_{n}=-\frac{2 \pi}{\Phi_{0}} \partial \epsilon_{n} / \partial \varphi$. More specifically, we focus on the so-called long diffusive junction limit where the length $l$ of the junction is greater than the superconducting coherence length in the normal metal $\xi=\sqrt{\hbar D / \Delta}$, with $D$ the diffusion coefficient in the normal metal. The Andreev spectrum exhibits a phasedependent minigap as shown in fig.1. The minigap only depends on the properties of the normal metal since its amplitude at $\varphi=0$ is $E_{g}=3.1 E_{T h}$ where the Thouless energy $E_{T h}=\hbar / \tau_{D}$ is the energy associated with the diffusion time $\tau_{D}=l^{2} / D$ [30-32]. As measured by tunnel spectroscopy [33], in the continuous spectrum limit, the minigap closes at $\varphi=\pi$ and reads:

$$
E_{g}(\varphi)=E_{g}|\cos (\varphi / 2)| \text {. }
$$

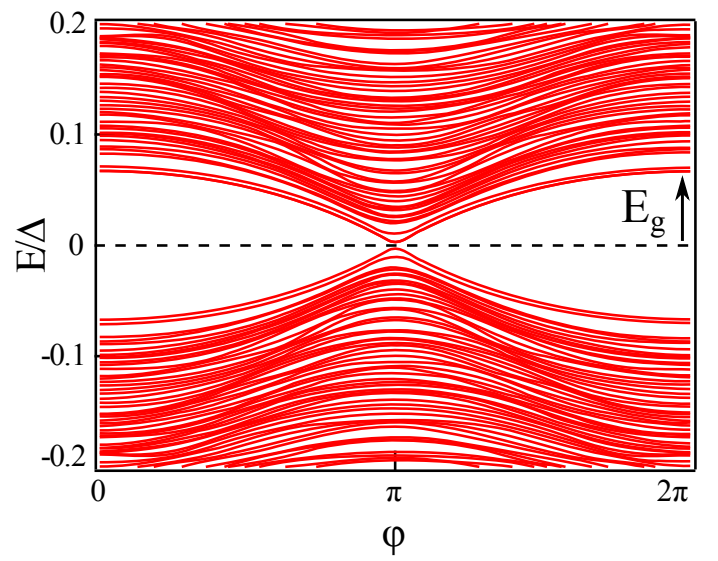

FIG. 1: Typical Andreev spectrum of a long SNS junction in the diffusive regime. Note the phase dependence and the $2 \pi$-periodicity of the spectrum, and the opening of the minigap. Spectrum is from [14].

We are interested in probing the system close to its thermodynamic equilibrium. We have therefore designed a linear response experiment in which one measures both the non-dissipative $\chi^{\prime}$ and dissipative $\chi$ " current response functions of an NS ring [11, 12]. We measure this response by inductively coupling an NS ring to a multimode superconducting resonator, thereby implementing both an ac phase bias and an ac current detection at the resonator's eigen-frequencies.

We find that at low frequency the non-dissipative current response $\chi^{\prime}$ corresponds, as expected, to the phase derivative of the supercurrent flowing through the ring, thus revealing the current-phase relation. A more striking finding is the existence of a dissipative response $\chi$ ", revealing that the supercurrent exhibits thermal noise, as discussed in [12]. In that paper we analyzed the low frequency, high temperature dissipation induced by the thermal relaxation of the population of the Andreev levels and a good agreement was found with theoretical predictions obtained by solving the time dependent Keldysh-Usadel equations [13, 34]. Time dependent Usadel equations $[13,16,34]$ as well as numerical simulations [14] also predict another dissipation mechanism related to microwave-induced transitions within the Andreev spectrum. This second contribution being dominant at high frequency and low temperature showing up as an absorption peak at $\pi$ increasing as one goes deeper in the quantum regime $\left(\hbar \omega>E_{g}>k_{B} T\right)$. Even if some indications of this second type of dissipation were found in [13] in the low temperature regime, the experiments were difficult to analyze because of screening effects. In this paper we present a complete quantitative analysis of experimental data over a wide frequency and temperature range on two different samples and we show how dissipation moves from one type to the other, in good agreement with these numerical and theoretical results 
$[14,16]$.

The paper is organized as follows: in the first section we describe the experimental setup used to probe the dynamics of Andreev bound states over a wide frequency range. In the second section we give an overview of our experimental results. The complete analysis is presented in the third section.

\section{EXPERIMENTAL SETUP}

The experiment consists in inductively coupling an NS ring to a multimode $\lambda / 4$ strip-line superconducting resonator operating between $190 \mathrm{MHz}$ and $16 \mathrm{GHz}$. The aim is to determine the complex magnetic susceptibility $\chi(\varphi, \omega, T)=\chi^{\prime}(\varphi, \omega, T)+i \chi^{\prime \prime}(\varphi, \omega, T)$ of the ring which relates the ac current response $I(\varphi, \omega, T)$ to the ac flux through the loop $\delta \phi_{a c} \exp -i \omega t$ in the linear response regime. The frequency $\omega$ is restricted to the successive resonances of the resonator $\omega_{n}$. The dc superconducting phase difference $\varphi$ at the boundaries of the $\mathrm{N}$ wire is imposed by a magnetic flux $\Phi$ created by a magnetic field perpendicular to the ring plane. The ac flux is generated by the ac current in the resonator. A similar technique based on single mode resonators was already used for the investigation of short Josephson junctions embedded in superconducting rings [35-37]. More related to the present work, the impedance of an array of SNS dc SQUIDS was measured using a superconducting multimode resonator [38]. This work focused on the temperature dependence of the effective inductive and resistive components of the SNS SQUIDs whereas our work focuses instead of the phase dependence which bares most of the signature of the dynamics of Andreev states $[14,16]$.

\section{Principle of the experiment}

The magnetic susceptibility of the NS ring $\chi$ modifies the inductance $L_{r}$ of the resonator and thus the eigenfrequencies $\omega_{n}=(2 n+1) \sqrt{1 / L_{r} C}$ and quality factors $Q_{n}$. The induced variations of resonance frequency and quality factor are related to the variations of the real and imaginary components $\Delta L_{r}^{\prime}$ and $\Delta L_{r}^{\prime \prime}$ of $L_{r}$ according to $\frac{\Delta \omega_{n}}{\omega_{n}}=-\frac{\Delta L_{r}^{\prime}}{2 L_{r}}$ and $\Delta\left(\frac{1}{Q_{n}}\right)=\frac{\Delta L_{r}^{\prime \prime}}{L_{r}}$.

This leads to the following relations between the measured susceptibility $\chi_{m}=\chi_{m}^{\prime}+i \chi_{m}^{\prime \prime}$ and the perturbation of the resonator's eigenmodes $\Delta \omega_{n}$ and $\Delta\left(\frac{1}{Q_{n}}\right)$ :

$$
\begin{gathered}
\chi_{m}^{\prime}=-2 \frac{L_{r}}{L_{c}^{2}} \frac{\Delta \omega_{n}}{\omega_{n}} \\
\chi_{m}^{\prime \prime}=\frac{L_{r}}{L_{c}^{2}} \Delta\left(\frac{1}{Q_{n}}\right)
\end{gathered}
$$

$L_{c}$ is the coupling inductance which is the part of the loop in parallel with the SNS junction, see fig.2. Due to screening of the applied flux by the finite geometrical inductance of the loop $L_{l}=L_{c}+L_{N}$, complex susceptibilities $\chi$ and $\chi_{m}$ are related through $\chi_{m}=\chi /\left(1-L_{l} \chi\right)$. The measured susceptibilities $\chi_{m}^{\prime}$ and $\chi_{m}^{\prime \prime}$ are therefore related to the intrinsic susceptibilities $\chi^{\prime}$ and $\chi^{\prime \prime}$ according to:

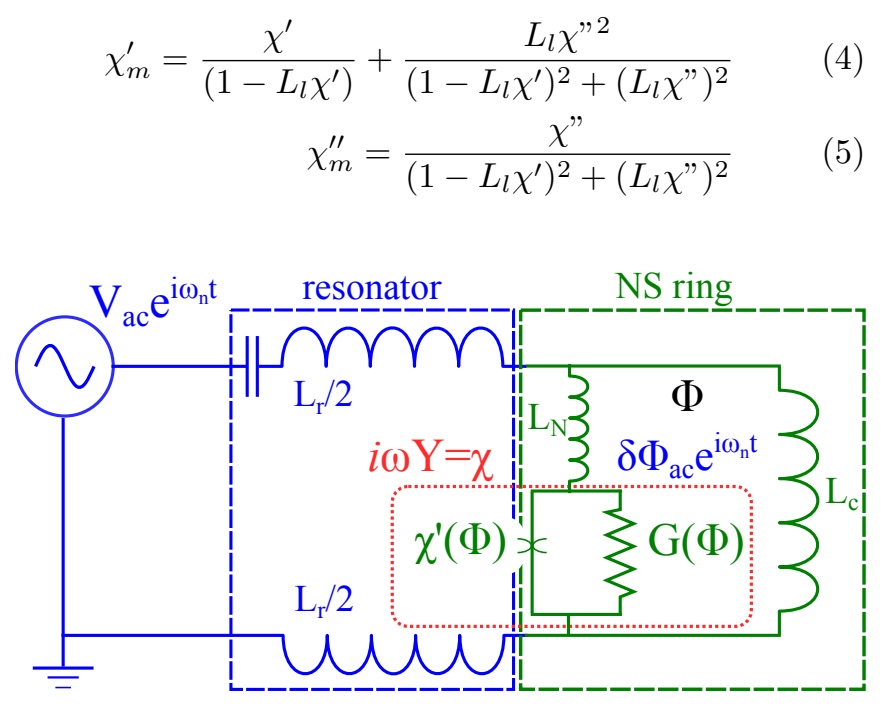

FIG. 2: Schematic representation of the NS ring inserted in the resonator. The resonator has an inductance $L_{r} . L_{c}$ and $L_{N}$ are respectively the coupling inductance and geometrical inductance of the normal wire, the total geometrical inductance of the NS ring being $L_{l}=L_{c}+L_{N}$. $G(\Phi)=\chi^{\prime \prime}(\Phi) / \omega$ is the dissipative component of the admittance of the NS ring, $\chi^{\prime}(\Phi)$ is its non-dissipative part.

When both $L_{l} \chi^{\prime} \ll 1$ and $L_{l} \chi^{\prime \prime} \ll 1$, these flux screening corrections are negligible, so that $\chi_{m}^{\prime}=\chi^{\prime}$ and $\chi_{m}^{\prime \prime}=\chi^{\prime \prime}$. Outside of this regime, [11, 39], screening leads to a dc flux rescaling and to hysteresis at low temperature when the parameter $\beta(T)=L_{l} \chi_{J}(T) \geq 1$. We have investigated a range of parameters where both $L_{l} \chi^{\prime}$ and $L_{l} \chi^{\prime \prime}$ are smaller than 1 but not necessary very small. In the latter case, these screening corrections must be properly taken into account in order to compare our results to theoretical predictions. We also note that the analysis of the dissipative response is more delicate than the non-dissipative one since $\chi^{\prime \prime}$ is mixed with $\chi^{\prime}$ within first order of $L_{l} \chi^{\prime}$.

These screening effects have led us to design samples of different size which enable the exploration of both regimes of low temperature and high frequency as well as the opposite regime of high temperature and low frequency. Experimentally we have no access to the phase independent components of $\chi_{m}$ which would require a very accurate comparison of the resonances with and without the sample. We measure instead accurately the phase dependence of $\chi_{m}^{\prime}$ and $\chi_{m}^{\prime \prime}$. Screening corrections on the phase dependent component of $\chi^{\prime \prime}$ increase drastically with frequency even at high temperature for which 
$L_{l} \chi^{\prime} \ll 1$. As discussed in the following, this leads to a dependence in the measured dissipative response entirely due to screening denoted $\chi_{s}^{\prime \prime}$. We will show that we can take advantage of this spurious phase dependence to determine the phase independent value of $\chi^{\prime \prime}$ which cannot be determined otherwise.

\section{Sample fabrication}

The linear response is measured by inserting an NS ring in a resonator (see fig.3). The resonator consists of two parallel superconducting $\mathrm{Nb}$ meander lines $(1 \mu \mathrm{m}$ thick, $2 \mu \mathrm{m}$ wide, $20 \mathrm{~cm}$ long, and $4 \mu \mathrm{m}$ apart) patterned on a sapphire substrate. The NS ring connects the two lines at one end of the resonator, turning it into a $\lambda / 4$ line with a fundamental frequency of $190 \mathrm{MHz}$, and harmonics 380 $\mathrm{MHz}$ apart. One of these lines is weakly coupled to a RF generator via a small on-chip capacitance whose value is adjusted in order to preserve the high $\mathrm{Q}$ of the resonances (of the order of 10000), the other one is grounded. The high $\mathrm{Q}$ factors enable detection of variations as small as $10^{-8}$, thus providing very accurate ac impedance measurements of mesoscopic objects. To make the NS rings, an Au wire is first fabricated by e-beam lithography and deposition of high purity gold $99.9999 \%$ (Sample A), 99.999 \% (Sample B). Since sapphire is an insulating substrate, we get rid of charging effects using a conducting espacer (300Z from Showa Denko Europe GmbH) over the usual PMMA/MAA resist. The $\mathrm{S}$ part is deposited in a second alignment step by sputtering of a $\mathrm{Pd} / \mathrm{Nb}$ bilayer $(6 \mathrm{~nm} \mathrm{Pd}, 100 \mathrm{~nm} \mathrm{Nb})$. The ring is connected to the $\mathrm{Nb}$ resonator in a subsequent step, using ion-beam assisted deposition of a tungsten wire in a focused ion beam (FIB) microscope. This process creates a good superconducting contact between the resonator and the $\mathrm{Pd} / \mathrm{Nb}$ part of the ring. The $6 \mathrm{~nm}$-thick $\mathrm{Pd}$ buffer layer ensures a good transparency at the NS interface, as demonstrated by the amplitude of the normal state conductance and critical current measured with dc transport measurements on control SNS junctions which have the same geometric properties and are fabricated simultaneously. Sample A has a critical current and loop inductance which are much larger than those of sample B. It is adequate for the investigation of the high temperature and low frequency regimes but not the low temperature and high frequency regime because of screening effects. In contrast sample B has a much smaller critical current and loop inductance and thus has negligible screening effects. This sample is therefore more adapted for the high frequency and low temperature regimes. For sample A, the $\mathrm{Au}$ wire is 0.3 micron wide, $50 \mathrm{~nm}$ thick and with a 1 micron long part that is not covered with $\mathrm{Pd} / \mathrm{Nb}$. The normal state resistance measured on a co-evaporated control sample is $1 \Omega$. The loop inductance is estimated to be $L_{l}=10 \pm 1 \mathrm{pH}$. The Au wire in sample B is $70 \mathrm{~nm}$ wide, $30 \mathrm{~nm}$ thick and 1.5 micron long. The estimated normal state resistance is $10 \Omega$, the loop inductance is $3 \pm 0.3 p H$.
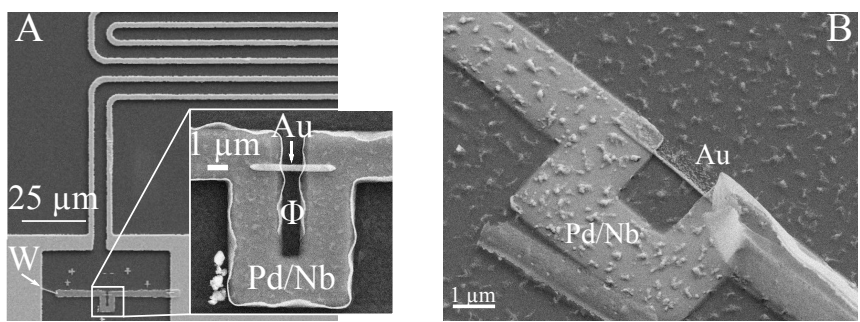

FIG. 3: The linear response is measured by inserting an NS ring in a resonator. Left: An NS ring is inserted in the middle of a $\lambda / 4$ multimode resonator. A FIB-deposited $\mathrm{W}$ wire ensure a good connection between the ring and the resonator. The insert shows a close-up view of sample A. Right: Micrograph of sample B.

\section{OVERVIEW OF THE EXPERIMENTAL RESPONSE}

As shown in fig. 4 and 5 we find a rich evolution of the phase dependence $\chi_{m}(\varphi)$ with temperature and frequency. At the lowest accessible frequency $\left(f_{0}=\right.$ $190 \mathrm{MHz})$ and high temperature $\left(T=1.2 \mathrm{~K} \simeq 17 \mathrm{E}_{\mathrm{Th}}\right)$, the non-dissipative component of $\chi_{m}$ measured on sample $\mathrm{A}$, displays a cosine phase dependence which is the derivative of the usual Josephson current-phase dependence, sinusoidal for $T \gtrsim E_{g}(\varphi=0)$ [9] with a decreasing amplitude as the temperature increases. The signal becomes highly non-sinusoidal at high frequency with a local maximum around $\varphi=0$ and a sharper phase dependence around $\pi$. This is a sign of a great harmonic content with in particular an important contribution of the second harmonic. Correlatively, the dissipative component $\chi_{m}^{\prime \prime}(\varphi)$ strongly depends on frequency. The second harmonic clearly dominates at low frequency and displays a sharp dip at $\pi$. At high frequency instead, this phase dependence evolves with the emergence a sharp peak at $\pi$. The amplitude of this dissipation peak increases at low temperature as shown on fig.5. These data are the signature of large non-adiabatic contributions to the phase-dependent response of NS rings which will be analyzed in detail in the following. On sample A, one needs to consider screening corrections distorting the phase dependence of $\chi_{m}^{\prime}$ and $\chi_{m}^{\prime \prime}$ at low temperature as will be discussed in the next section. In contrast these corrections are negligible on sample B for which one can safely assume that $\chi=\chi_{m}$ over the whole temperature and frequency range investigated. Data shown in fig.5 at 15.5 GHz show similar phase dependence for $\chi^{\prime}$ and $\chi^{\prime \prime}$ with peaks at $\pi$ increasing at low temperature. 


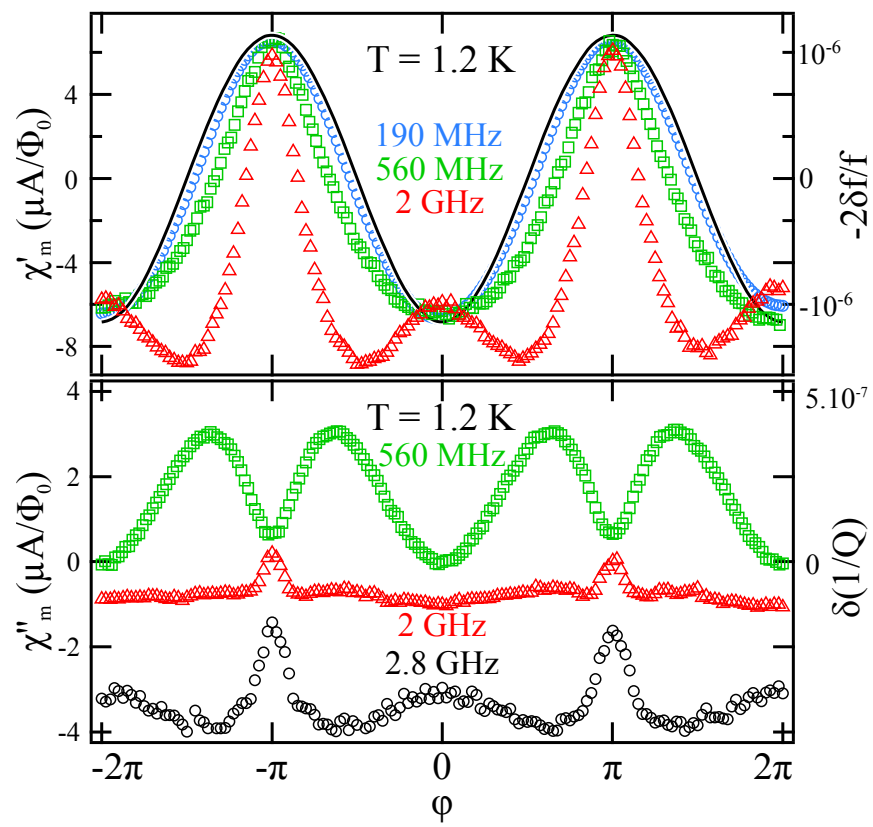

FIG. 4: Evolution of the experimental response with frequency at high temperature. Top: Non-dissipative $\chi_{m}^{\prime}$ and Bottom: dissipative $\chi_{m}^{\prime \prime}$ response at several frequencies and $T=1.2 \mathrm{~K}$. Note that the amplitude between 0 and $\pi$ of $\chi_{m}^{\prime}$ does not depend on frequency whereas its harmonic contents does. For the sake of clarity, $\chi_{m}^{\prime \prime}(2 \mathrm{GHz})$ and $\chi_{m}^{\prime \prime}(2.8 \mathrm{GHz}$ have been arbitrarily offset.

\section{ANALYSIS}

In the following we compare these results with theoretical expectations based on the linear response calculation relating the susceptibility to the phase-dependent Andreev eigenstates derived from the diagonalisation of the Bogoliubov-de Gennes Hamiltonian of a diffusive SNS junction $[12,14]$ according to:

$$
\begin{aligned}
& \chi(\omega, \varphi, T)=\frac{\partial I_{J}}{\partial \varphi}-\sum_{n} i_{n}^{2} \frac{\partial f_{n}}{\partial \epsilon_{n}} \frac{i \omega}{\tau_{i n}^{-1}-i \omega}- \\
& \sum_{n, m \neq n}\left|J_{n m}\right|^{2} \frac{f_{n}-f_{m}}{\epsilon_{n}-\epsilon_{m}} \frac{i \hbar \omega}{i\left(\epsilon_{n}-\epsilon_{m}\right)-i \hbar \omega+\hbar \gamma_{N D}}
\end{aligned}
$$

$J_{n m}$ is the matrix element of the current operator between the Andreev eigenstates $|n\rangle$ and $|m\rangle$ of energies $\epsilon_{n}(\varphi)$ and $\epsilon_{m}(\varphi), f_{n}$ is the Fermi Dirac function at energy $\epsilon_{n}$.

The first term is the zero frequency susceptibility of the ring, $\chi(\omega=0)=\partial I_{J} / \partial \varphi$. We call it Josephson contribution $\chi_{J}$. The second and third terms only exist at finite frequency and describe the non-adiabatic, dynamical responses due respectively to the relaxation of the populations $\chi_{D}$, and to the transitions between the levels induced by microwave photons emission or absorption $\chi_{N D}$, the quantities $1 / \tau_{i n}$ and $\gamma_{N D}$ being respectively the diagonal and non-diagonal relaxation rates of the system determined by its interaction with its thermodynamic environment. Dissipation is described by the imaginary components of the diagonal and non-diagonal susceptibilities $\chi_{D}^{\prime \prime}$ and $\chi_{N D}^{\prime \prime}$. Their different phase dependence reveal their different physical origins as sketched in fig.6. $\chi_{D}^{\prime \prime}$ is proportional to the square of the single level current and must be zero at phases multiples of $\pi$. In contrast, $\chi_{N D}^{\prime \prime}(\varphi)$ is determined by the interplay of the phasedependent non-diagonal elements of the current operator, the occupation of the levels, and the presence of the minigap in the density of states. Its phase dependence therefore strongly depends on the relative amplitude of $\hbar \omega, k_{B} T$ and $E_{g}$. In addition, $\chi_{N D}^{\prime \prime}(\varphi)$ has a phaseindependent part that is expected to ultimately gives the normal conductance when no trace of coherence is left. In the regime of high temperature and low frequency the non-diagonal elements of the current operator lead to a phase dependence of $\chi_{N D}^{\prime \prime}(\varphi)$ opposite to $\chi_{D}^{\prime \prime}(\varphi)$ whereas at low temperature dissipation is depressed except at $\pi$ due to the minigap, leading to a dissipation peak at $\pi$ in the phase-dependent part of the dissipation. These phase and frequency dependence are discussed in more details in Appendix A. The analysis of our experimental results reveals these contributions and the physical mechanisms underpinning them. We first discuss data on sample A that reveals the adiabatic response as well as the dynamic response in the low frequency and high temperature regime. We also determine the phase-independent contribution to the conductance that is revealed through flux screening effects. In contrast, data on sample B (for which screening corrections are negligible) show unambiguously a dissipation peak at $\pi$ related to the presence of the minigap.

\section{Notations}

We use the following notations: $\delta_{\varphi_{1}-\varphi_{2}} \xi=\xi\left(\varphi_{1}\right)-$ $\xi\left(\varphi_{2}\right)$ and $\delta \xi$ is the maximum absolute amplitude of the phase dependence of $\xi$ which stands for $\chi^{\prime}, \chi^{\prime \prime}, \chi_{m}$ or $\chi_{m}^{\prime \prime}$.

\section{Adiabatic response $\chi_{J}$}

For frequencies such that $\omega \ll \gamma_{D} \leq \gamma_{N D}$, the diagonal and non-diagonal contributions are negligible, so $\chi^{\prime}(\varphi)=\chi_{J}(\varphi)$ yields the phase derivative of the currentphase relation at equilibrium. The phase dependence of $\chi^{\prime}$ is obtained from $\chi_{m}^{\prime}(\varphi)$ after correction of the self inductance effects via:

$\delta_{\varphi-0} \chi_{m}^{\prime}=\chi_{m}^{\prime}(\varphi)-\chi_{m}^{\prime}(0)=\frac{\chi^{\prime}(\varphi)}{1-L_{l} \chi^{\prime}(\varphi)}-\frac{\chi^{\prime}(0)}{1-L_{l} \chi^{\prime}(0)}$

using that $\chi^{\prime}(\pi)=-\chi^{\prime}(0)=\chi_{J}(\pi)$. 

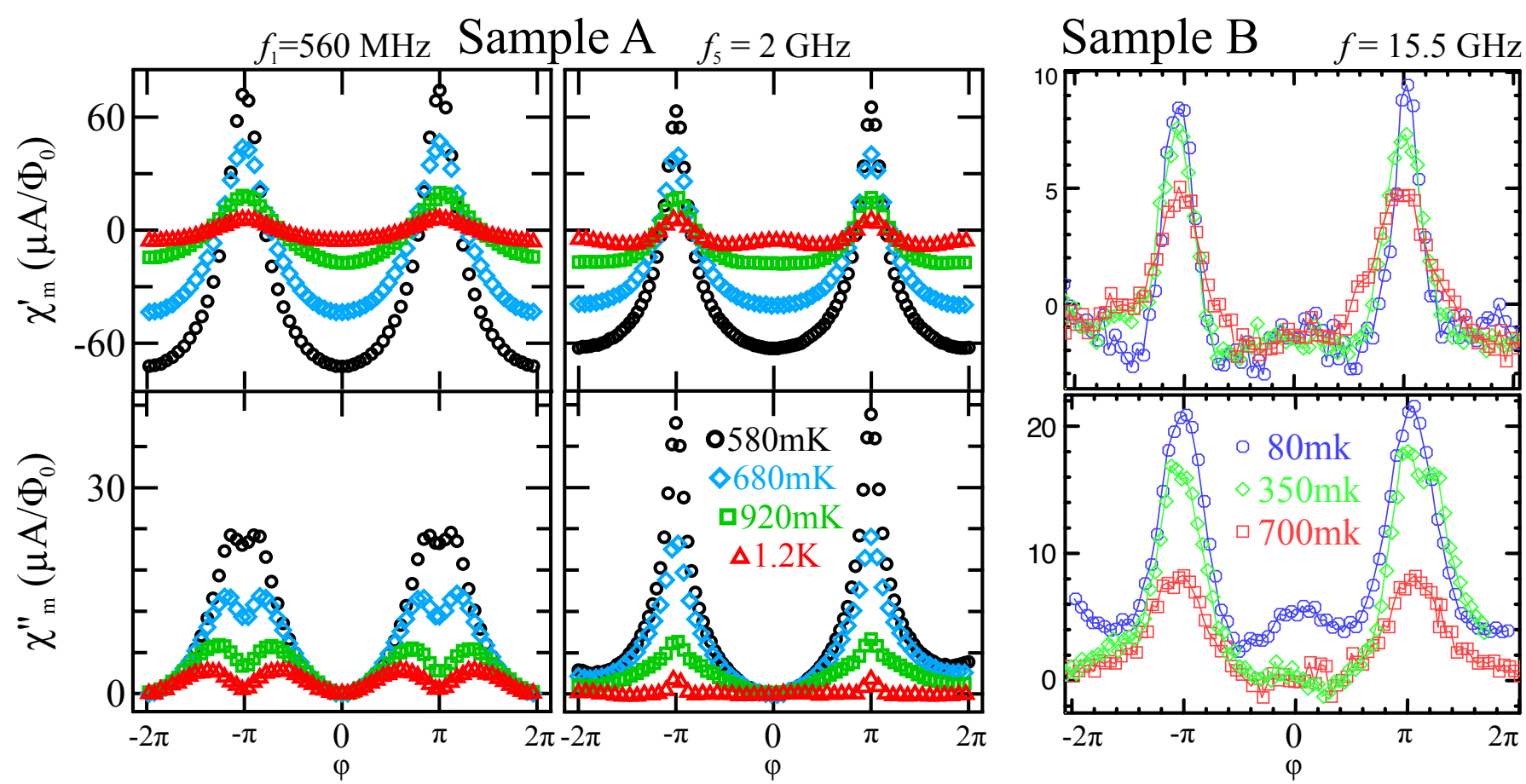

FIG. 5: Evolution of the measured response with temperature. Evolution of $\chi_{m}^{\prime}(\varphi)$ (top) and $\chi_{m}^{\prime \prime}(\varphi)$ (bottom) with temperature. Sample A: $f_{1}=560 \mathrm{MHz}<\mathrm{E}_{\mathrm{Th}} / \mathrm{h}$ (left) and $f_{5}=2 \mathrm{GHz} \gtrsim \mathrm{E}_{\mathrm{Th}} / \mathrm{h}$ (right). Sample B: $\mathrm{f}=15.5 \mathrm{GHz}$.Curves have been shifted vertically so that $\chi_{m}^{\prime}(\varphi=\pi)+\chi_{m}^{\prime}(\varphi=0)=0$ and $\chi_{m}^{\prime \prime}(\varphi=0)=0$. In both cases the calibration of the susceptibility is computed from the perturbation of the resonances using expressions 2 and 3.

For the lowest eigen-frequency of the resonator, see fig.7, $\chi^{\prime}(\varphi)$ is well described by a cosine and its amplitude is simply related to the critical current by $\delta_{\pi-0} \chi^{\prime}=$ $\frac{4 \pi}{\Phi_{0}} I_{c}=2 \chi_{J}(\pi)$. Noting that $\chi_{D}^{\prime}$ does not modify $\delta_{\pi-0} \chi^{\prime}$, we find (see fig.7) that this relation between the amplitude of $\chi^{\prime}$ and $I_{c}$ holds in the whole temperature range investigated where $k_{B} T \gg E_{T h} . \delta_{\pi-0} \chi^{\prime}(T)$ follows the expected exponential decay of the Josephson critical current $I_{c}(T)=I_{J}(0) \exp \left(-k_{B} T / 3.6 E_{T h}\right)[40]$. Fitting this dependence yields $E_{T h} / k_{B}=71 \pm 2 \mathrm{mK}$. These results agree with the temperature dependence of the switching current of a current-biased control junction that has similar geometrical characteristics. The analysis of the amplitude of $\delta_{\pi-0} \chi^{\prime}$ in the low frequency regime thus allows to perfectly characterize the junction and yields the total minigap width $2 E_{g}(0) / h=9 \mathrm{GHz}$ and the corresponding diffusion time across the junction $\tau_{D}=0.1 \mathrm{~ns}$.

Main contribution at low frequency and high temperature: relaxation of the populations of Andreev states.

In this paragraph we focus on the diagonal susceptibility which is due to the Debye-like relaxation of the occupation of the Andreev levels driven out-of-equilibrium by the ac phase excitation [34]. This is the first non- adiabatic contribution observed at modererate frequencies when frequency is of the order of the relaxation time of the phase-dependent populations $\tau_{i n}$. According to expression 6 , frequency and phase dependence simply factorize. The frequency dependence is described by the simple Debye relaxation law in $\omega \tau_{i n} /\left(1-i \omega \tau_{i n}\right)$ and the phase dependence of $\chi_{D}$ is therefore identical for both its real and imaginary components.

At these low enough frequencies so that the nondiagonal contribution to $\chi^{\prime}$ stays negligible, the most reliable way to access this phase dependence at temperatures above the Thouless energy is to subtract the adiabatic response to the susceptibility: $\chi_{D}^{\prime}(\varphi)=\chi^{\prime}(\varphi)-\chi_{J}(\varphi)$ (see fig.8.a) where $\chi_{J}(\varphi)$ is a simple cosine. The experimental determination of $\chi_{D}^{\prime \prime}(\varphi)$ is more delicate and needs to be restricted to the range of temperature and frequency where both screening $\chi_{s}^{\prime \prime}$ and $\chi_{N D}^{\prime \prime}$ contributions are negligible. An example is shown in fig. 8 at high temperature $(\mathrm{T}=1.2 \mathrm{~K})$ and low frequency $(\mathrm{f}=560 \mathrm{MHz})$ where the measured signal $\chi_{m}^{\prime \prime}$ is clearly dominated by $\chi_{D}^{\prime \prime}(\varphi)$. Indeed the phase dependence exhibits the characteristic shape of $\sum \partial f / \partial \epsilon_{n}$, typical of the relaxation of Andreev levels occupations. It is zero at 0 and $\pi$ as expected for the square of the single level current with a sharp dip at $\pi$ coming from the contribution of the Andreev levels close to the minigap [12]. We compare successfully this flux dependence with the Lempitski function $F_{U}(\varphi)$ [41] corresponding to the time-dependent Usadel equa- 


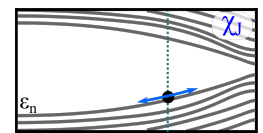

$\varphi_{\mathrm{dc}}+\varphi_{\mathrm{ac}}$
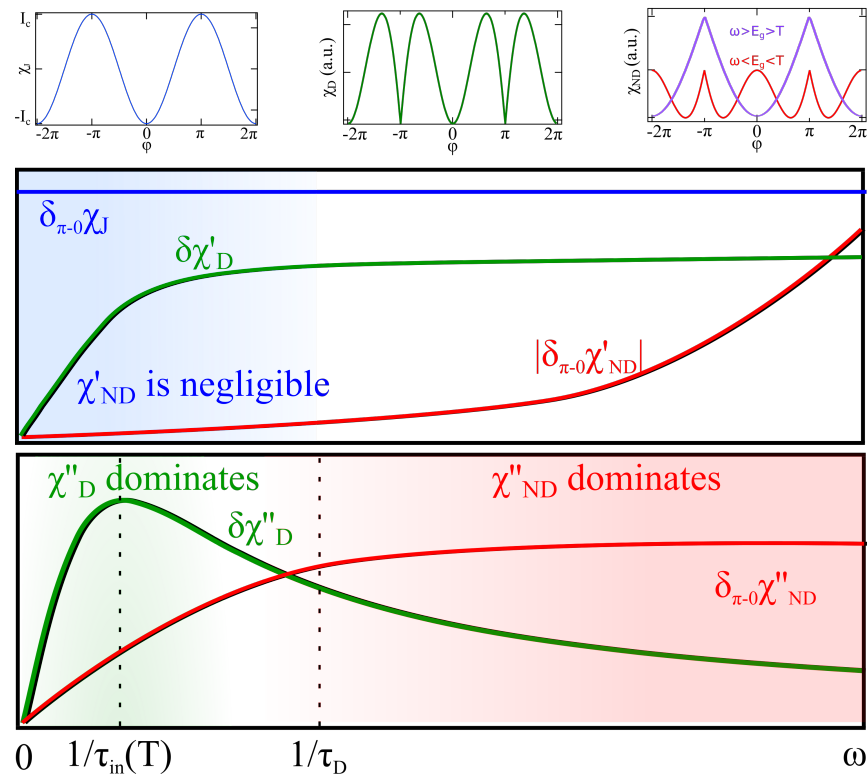

FIG. 6: Schematic frequency-evolution of the response. Top: Sketch of the physical mechanisms at the origin of the finite-frequency response: (Left:) adiabatic response $\chi_{J}$; (Middle:) relaxation of populations driven out-ofequilibrium by the finite-frequency phase-biasing, leading to $\chi_{D}^{\prime \prime}$; (Right:) microwave induced transitions across the minigap, leading to $\chi_{N D}^{\prime \prime}$. Middle: Phase dependence of each contribution. $\chi_{J}$ is a cosine when the equilibrium currentphase relation is purely sinusoidal, $\chi_{D}$ has almost half the periodicity of $\chi_{J}$. The phase dependence of $\chi_{N D}^{\prime \prime}$ depends on the temperature and frequency. At low temperature and high frequency, $\chi_{N D}^{\prime \prime}$ follows the minigap whereas at low frequency and high temperature $\chi_{N D}^{\prime \prime}$ has a phase dependence opposite to the one of $\chi_{D}^{\prime \prime}$. Bottom: Schematic frequency dependence of each contribution for the non-dissipative (Top) and dissipative (Bottom) responses. At low frequency, $\chi_{N D}^{\prime \prime}$ is negligible and $\chi^{\prime \prime}$ is dominated by $\chi_{D}^{\prime \prime}$ for $\omega \tau_{i n} \sim 1$. At high frequency $\chi^{\prime \prime}$ is dominated by $\chi_{N D}^{\prime \prime}$.

tions (see Appendix A and [13]). We also observed that this shape of $\chi_{D}^{\prime}(\varphi)$ is independent of temperature [12] for $k_{B} T \gg E_{g}$. The frequency dependence of $\delta \chi_{D}^{\prime}$ are shown in fig. 8 for different temperatures. They can be fitted by the expected $\frac{\left(\omega \tau_{i n}\right)^{2}}{1+\left(\omega \tau_{i n}\right)^{2}}$ which enables to determine the characteristic time $\tau_{i n}(T)$.

We find values of $\tau_{i n}$ varying between like $1 / T$ [42]. This temperature dependence is similar to the predicted $\mathrm{T}$ variation of the phase coherence time in a short diffusive wire when it is limited by electron-electron inelastic scattering [43-46]. However the prefactor in our experiment is more than 2 orders of magnitude smaller. This result points towards a different mechanism, possibly due to the thin $\mathrm{Pd}$ layer at the interface between the $\mathrm{Nb}$ and
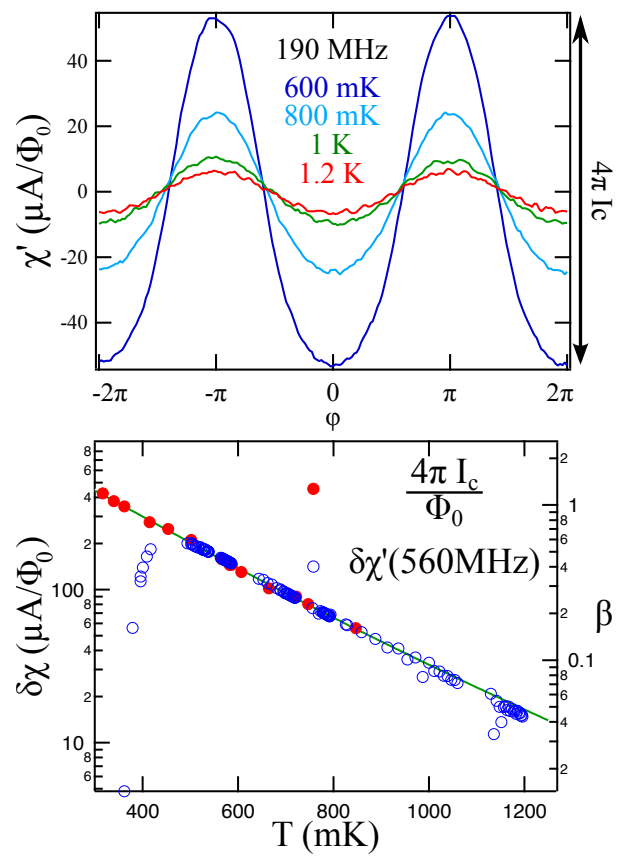

FIG. 7: Characterization of the ring at low frequency. Upper panel Phase-dependence of the measured non-dissipative response $\chi_{m}^{\prime}$ at $190 \mathrm{MHz}$ and several temperatures. At low frequency, the amplitude of $\chi_{m}^{\prime}$ is directly proportional to the ring's critical current: $\delta_{\pi-0} \chi^{\prime}=4 \pi I_{c} / \Phi_{0}$. Lower panel Temperature dependence in semi-log scale of the amplitude of the non-dissipative response of the ring at $560 \mathrm{MHz}, \delta \chi^{\prime}(f=560 \mathrm{MHz})$ (circles) and of the control sample using that $\delta \chi^{\prime}=4 \pi I_{c} / \Phi_{0}$ (full circles) along with its fit (solid line). $E_{T h}=71 m K$ is found.

Au yielding low energy sub-gap excitations.

We now return to the temperature dependence of $\chi_{D}^{\prime}$ resp. $\chi_{D}^{\prime \prime}$ shown in fig.9 at fixed frequency. They follow the expected $T^{-1} \frac{\omega^{2} \tau_{i n}^{2}}{1+\omega^{2} \tau_{i n}^{2}}\left(\right.$ resp. $\left.T^{-1} \frac{\omega \tau_{i n}}{1+\omega^{2} \tau_{i n}^{2}}\right)$. The $1 / T$ prefactor comes from the energy derivative of the Fermi distribution at $T \gg E_{g}$. Interestingly, this temperature dependence is much slower than the exponential decrease of the supercurrent. This result agrees with the predictions of Zhou and Spivak on the conductance of a SNS junction [34]. We note that a similar temperature dependence was also found for the fractional Shapiro steps also due to this relaxation of the population of the Andreev states [47-49].

\section{Main contribution at high frequency and low temperature: microwave-induced transitions between Andreev states.}

The aim of the following paragraph is to analyse the dissipation when frequency becomes larger than $1 / 2 \pi \tau_{\text {in }}$ and the contribution of induced transitions within the Andreev spectrum, $\chi_{N D}$, becomes important. The analysis of $\chi_{N D}^{\prime}$ is involved (see Appendix A) and we there- 

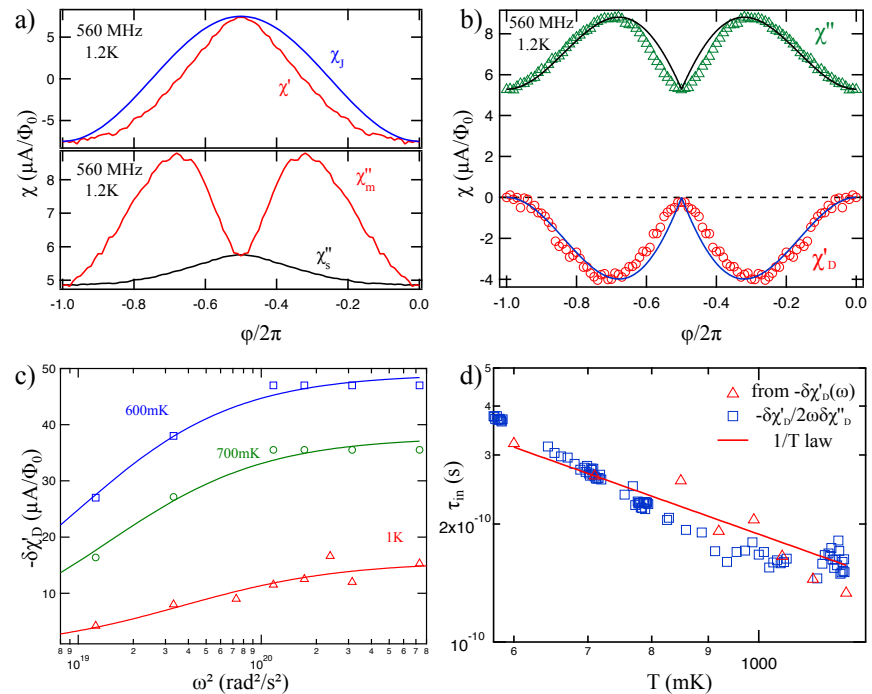

FIG. 8: Diagonal contribution to $\chi^{\prime}$ and $\chi$ ". a: Phase dependence at $T=1.2 \mathrm{~K}$ and $560 \mathrm{MHz}$ of: the adiabatic response $\chi_{J}$ and the finite frequency response $\chi^{\prime}$ (top panel), the measured dissipative response $\chi_{m}^{\prime \prime}$ and the screening contribution $\chi_{s}^{\prime \prime}$ (bottom panel). We note that screening effects are negligible on $\chi^{\prime}$ and therefore $\chi_{m}^{\prime} \simeq \chi^{\prime}$ at this temperature. b: At high temperature and for $f \lesssim E_{T h} / h$, experimental $\chi_{D}^{\prime}=\chi^{\prime}-\chi_{J}$ and $\chi^{\prime \prime}=\chi_{m}^{\prime \prime}-\chi_{s}^{\prime \prime}$ are found to be in very good agreement (within a factor 2) with theoretical prediction of Usadel equations given by the Lempitski function $F_{U}$ (solid lines). Theoretical predictions have been multiplied by an arbitrary factor so that their amplitude matches the experimental ones. c: Frequency dependence of $-\delta \chi_{D}^{\prime}$ : the maximum of $-\chi_{D}^{\prime}(\varphi)$, at different temperatures (symbols) compared to the theoretical prediction (see main text). d: Temperature dependence of $\tau_{i n}$ independently determined from the frequency dependence of $-\delta \chi_{D}^{\prime}$ (triangles) and from the ratio $\delta \chi_{D}^{\prime} /\left(\omega \delta \chi_{D}^{\prime \prime}\right)$ at $560 \mathrm{MHz}$ (squares) along with a $T^{-1}$ law (solid line).

fore focus on $\chi_{N D}^{\prime \prime}$. It can be noted however that $\chi_{m}^{\prime}$ is almost temperature independent up to $700 \mathrm{mK}$ at $15.5 \mathrm{GHz}$ (see fig.5) which shows that $\chi_{N D}^{\prime}$ is depressed at high frequency and low temperature and becomes much smaller than $\chi_{J}$. The amplitude of $\chi_{J}$ is estimated to be of the order of $55 \mu \mathrm{A} / \Phi_{0}$ at $T \lesssim E_{T h}$ for sample B.

We consider separately the high and low temperature regimes compared to the minigap which were respectively explored in sample A and sample B. On sample A, screening corrections are important and strongly distort the measured phase dependence. However these distortions can be exploited to determine the phase-independent component of $\chi^{\prime \prime}$. This is done by the determination of $\chi^{\prime \prime}(\pi)$ which is not simply equal to $\omega G_{N}$ but is found to vary with temperature in agreement with the predictions of $[14,16]$. The opposite regime of low temperature was explored using sample B and reveals the signature of the minigap. The very small amplitude of the signals measured did not allow to explore the high temperature regime on this second sample.
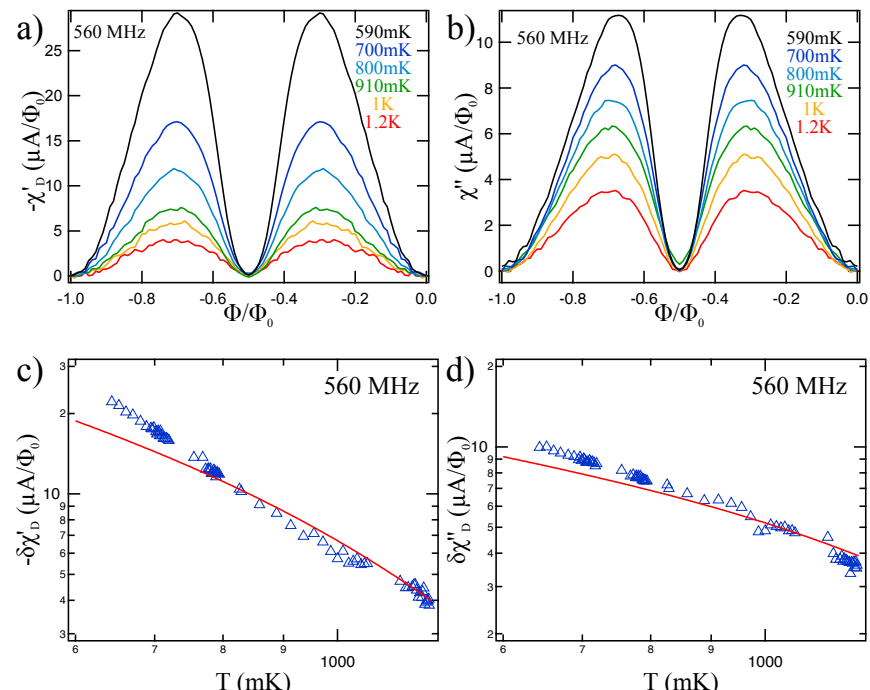

FIG. 9: Evolution of the diagonal contribution with temperature. Top: $-\chi_{D}^{\prime}$ and $\chi^{\prime \prime}$ at $f=560 \mathrm{MHz}$ and several temperatures. As seen from the phase dependence, $\chi^{\prime \prime}$ is dominated by $\chi_{D}^{\prime \prime}$ at this frequency. Bottom: Temperature dependence of the amplitude of $-\chi_{D}^{\prime}\left(\operatorname{resp} . \quad \delta \chi_{D}^{\prime \prime}\right)$ at several frequencies (symbols) along with the corresponding $T^{-1} \frac{\omega^{2} \tau_{i n}^{2}}{1+\omega^{2} \tau_{i n}^{2}}$ temperature dependence (resp. $T^{-1} \frac{\omega \tau_{i n}}{1+\omega^{2} \tau_{i n}^{2}}$ ) (solid lines). $\frac{\omega^{2} \tau_{i n}^{2}}{1+\omega^{2} \tau_{i n}^{2}}$ and $\frac{\omega \tau_{i n}}{1+\omega^{2} \tau_{i n}^{2}}$ are determined using the $T^{-1}$ fit of the experimental $\tau_{i n}(T)$ (fig. 8)

Temperature above the minigap: competition between $\chi_{D}^{\prime \prime}$ and $\chi_{N D}^{\prime \prime}$

We show that in this regime explored on sample A, not only the phase dependence of the non-diagonal contribution $\chi_{N D}^{\prime \prime}$ but also the absolute amplitude of $\chi^{\prime \prime}(\pi)$ can be revealed. We start from expression (5) relating the measured $\chi_{m}^{\prime \prime}$ to $\chi^{\prime}$ and $\chi^{\prime \prime}$ in the limit where $\chi^{\prime \prime} \ll \chi^{\prime}$ :

$$
\chi_{m}^{\prime \prime}=\frac{\chi^{\prime \prime}}{\left(1-L_{l} \chi^{\prime}\right)^{2}}
$$

This equation shows that even a constant (phase independent) $\chi^{\prime \prime}=\chi_{c}^{\prime \prime}$ will display a phase dependence given by $\chi_{s}^{\prime \prime}(\varphi)=\chi_{c}^{\prime \prime} /\left(1-L_{l} \chi^{\prime}(\varphi)\right)^{2}$ as shown in fig.10. This screening contribution needs to be determined in order to deduce the intrinsic $\chi^{\prime \prime}(\varphi)$. The protocol is described in detail in Appendix B. $\chi^{\prime \prime}(\pi)$ is extracted from the ratio $R_{m}=\delta_{\pi-0} \chi_{m}^{\prime \prime} / \delta_{\pi-0} \chi_{m}^{\prime}$. It is interesting that we could take advantage of screening to determine the absolute value of $\chi^{\prime \prime}$ at $\varphi=\pi$ and therefore the phase independent component of $\chi^{\prime \prime}$ which cannot be determined otherwise. Since the minigap closes at $\varphi=\pi$, with a density of states similar to the one of a normal metal, it could be expected that $\chi^{\prime \prime}(\pi)$ would be given by $\omega G_{N}$ and independent of temperature. As shown in fig.10, we find instead that $\chi^{\prime \prime}(\pi)$ increases above $G_{N}$ at low temperature. Within our experimental uncertainty, the 

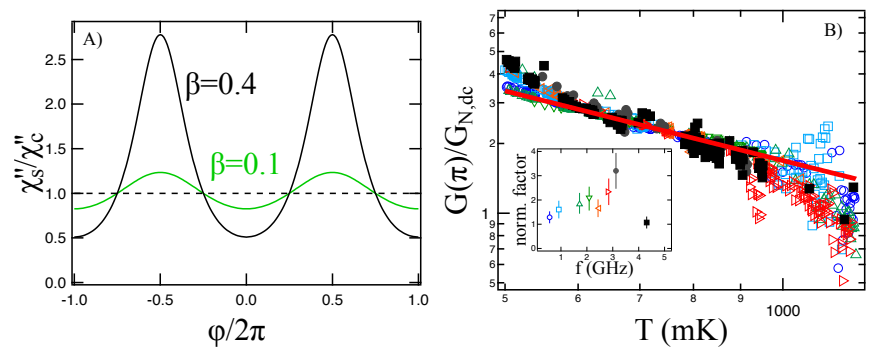

FIG. 10: Determination of $\chi^{\prime \prime}(\pi, T, \omega)$. A) Phasedependent dissipative response $\chi_{s}^{\prime \prime}$ due to screening. $\chi_{s}^{\prime \prime}=$ $\chi_{c}^{\prime \prime} /\left(1-L_{l} \chi^{\prime}(\varphi)\right)^{2}$ is shown for $\beta=0.4$ (black) and $\beta=0.1$ (green) where a simple $L_{l} \chi^{\prime}(\varphi)=-\beta \cos (\varphi)$ has been used. B) The temperature dependence of $G(\pi)$ is obtained from the ratio between the amplitudes of $\chi_{m}^{\prime \prime}$ and $\chi_{m}^{\prime}$ as explained in Appendix B. (Different symbols correspond to the different frequencies shown in the inset). A good agreement with a $1 / T$ law (solid line) is observed in a regime of intermediate temperatures. Data have been renormalized so that $G(\pi)$ at $1.2 \mathrm{~K} \simeq G_{N, d c}$ with $G_{N, d c}$ the conductance of the control sample. The frequency-dependent normalization factor is shown in the inset.

value of $G(\pi)$ above $1 \mathrm{~K}$ is similar to the normal state conductance of the control sample $G_{N}=0.9 \pm 0.2 \mathrm{~S}$ and $G(\pi, T)=\chi^{\prime \prime}(\pi, T) / \omega \propto T^{-1} G(\pi)$. This important increase of $G(\pi)$ up to values much larger than $G_{N}$ at low temperatures is surprising. It is in qualitative agreement with recent predictions $[13,14,16]$. We understand this effect as being related to phase-dependent correlations in the Andreev spectrum extending to energy scales much larger than the minigap (see Appendix A) with a special contribution of matrix elements $\left|J_{-n, n}\right|^{2}$ coupling electron-hole symmetric Andreev levels. Moreover it can be argue that this increase in dissipation should be taken into account when considering the Joule power dissipated by a junction to determine the temperature at which hysteresis in the I-V characteristic appears as done in $[50,51]$

This determination of $\chi^{\prime \prime}(\pi, T)$ is essential to reconstruct the intrinsic phase dependence of $\chi^{\prime \prime}$ from $\chi_{m}^{\prime \prime}(\varphi)$ according to:

$\chi^{\prime \prime}(\varphi)=(1-\beta(\varphi))^{2}\left(\chi_{m}^{\prime \prime}(\varphi)-\chi_{m}^{\prime \prime}(\pi)+\frac{\chi^{\prime \prime}(\pi)}{\left(1-\chi^{\prime}(\pi) L_{l}\right)^{2}}\right)$

where $\beta(\varphi)=L_{l} \chi^{\prime}(\varphi)$.

The resulting phase dependence of $\chi^{\prime \prime}$ are shown in fig.11 for different frequencies and temperatures. One can see the crossover between the low frequency regime where $\chi_{D}^{\prime \prime}$ (characterized by a minimum of dissipation at $\pi$ ) is dominant and the high frequency regime, $\omega \tau_{\text {in }} \gg 1$, where $\chi_{D}^{\prime \prime}$ becomes negligible compared to $\chi_{N D}^{\prime \prime}$, and the phase-dependent part of the dissipation starts to peak at $\pi$ with a peak amplitude increasing with frequency. This evolution in the phase dependence reflects the competition between the contribution of the diagonal elements of the current operator (minimum at $\pi$ due to the can- cellation of single level currents) and the contribution of the non-diagonal matrix elements of the current operator which, in contrast, are maximum at $\pi$ where Andreev levels anti-cross. In this range of temperature, $T \gg E_{T h}$ the contribution of the phase-dependent minigap is still negligible and the phase dependence of $\chi_{N D}^{\prime \prime}$ is essentially determined by the $\left|J_{n m}\right|^{2}$ and is opposite in sign to the phase dependence of $\chi_{D}$ (see appendix A).

The amplitude of the phase-dependent modulation of $\delta \chi_{N D}^{\prime \prime}$ also follows a $1 / T$ law like $\chi^{\prime \prime}(\pi)$ stemming from the temperature dependence of the Fermi functions differences $f_{n}-f_{m}$. This $1 / \mathrm{T}$ dependence, similar to the temperature dependence of $\chi_{D}$ in the same $\mathrm{T}$ range $\left(T \gg E_{g}\right)$, is much slower than the exponential $\mathrm{T}$ decay of the Josephson current, demonstrating that the dynamical response is much more robust than the critical current to temperature.

\section{Low temperature and high frequency: signature of the minigap}

We now consider high frequency data measured on sample B. Since for this sample screening corrections are completely negligible with $\beta \leq 0.03$, the measured response coincides with the intrinsic one down to very low temperature compared to the minigap. As shown in the panel B of fig. 5 and fig.12 the phase dependence of $\chi^{\prime \prime}$ displays a sharp dissipation peak at $\pi$. The phase dependence of $\chi^{\prime \prime}(\varphi)$ is shown in fig. 12 for different frequencies of the order or larger than the minigap of sample B estimated as: $2 E_{g} / h=5 \mathrm{GHz}$. This phase dependence as well as the amplitude of the dissipation peaks at $\pi$ are found to be only slightly frequency dependent. This result is in agreement with the expected saturation of $\delta_{\pi-0} \chi^{\prime \prime}$ at frequencies greater than the Thouless energy close to the estimated value of $2 G_{N} E_{g} / \hbar$, see Appendix $\mathrm{A}$ and [14]. We find a saturation at $\delta_{\pi-0} \chi^{\prime \prime} \simeq 15 \mu \mathrm{A} / \Phi_{0}$ whereas $2 G_{N} E_{g} / \hbar=12 \mu \mathrm{A} / \Phi_{0}$.

The temperature dependence of $\delta_{\pi-0} \chi^{\prime \prime}$ is shown fig.12 for different frequencies above and below $E_{g}$. It varies as $1 / T$ and saturates at a temperature below $0.4 K$ for 15 and $16 \mathrm{GHz}$ whereas no clear saturation can be detected at $3 \mathrm{GHz}$. Our data are compared with the theoretical expectation neglecting the phase dependence of matrix elements of the current operator:

$$
\begin{aligned}
& \delta_{\pi-0} \chi^{\prime \prime}(T)=G_{N} \int_{-E_{g}}^{+E_{g}}(f(E+\hbar \omega)-f(E)) d E= \\
& \frac{G_{N} k_{B} T}{\hbar}\left(-\log \frac{e^{\frac{\omega-E g}{T}}+1}{e^{\frac{E g+\omega}{T}}+1}+E_{g} / k_{B} T\right)
\end{aligned}
$$

The temperature dependence of $\delta_{\pi-0} \chi^{\prime \prime}(T)$ is strongly related to the behavior of the Fermi function difference $\delta(T, \omega)=f(E)-f(E+\hbar \omega) . \quad \delta(T, \omega)$ exhibits a $\mathrm{E} / \mathrm{T}$ dependence at high temperature compared to $\hbar \omega$ and saturates at low temperature of the order of $\hbar \omega / 4$ to a value equal to $4 E / \hbar \omega$. 

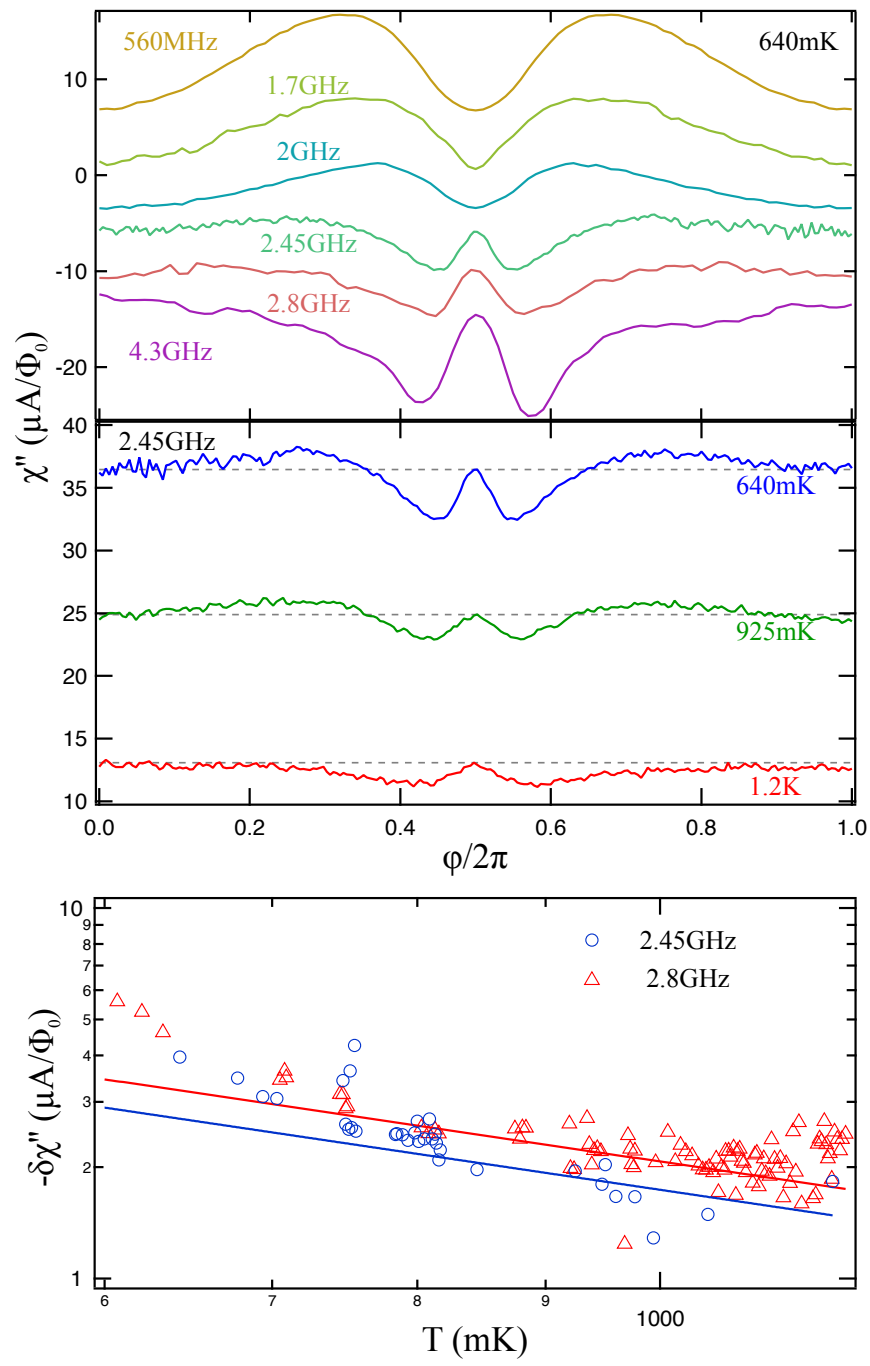

FIG. 11: Phase and temperature dependences of the dissipation at $T>E_{g} / k_{B}$. Top panel: evolution of the phase dependence of $\chi^{\prime \prime}$ with frequency at $640 \mathrm{mK}$ from top to bottom $0.56,1.7,2.2 .45,2.8,4.3 \mathrm{GHz}$, (curves are arbitrarily shifted from one another). Middle panel: evolution of $\chi^{\prime \prime}(\varphi)$ for different temperatures at $2.45 \mathrm{GHz}$, (in contrast with upper panel there is no added offset between the curves). Bottom panel: temperature dependence of $\delta \chi^{\prime \prime}$, the continuous lines corresponds to a $1 / \mathrm{T}$ decay.

In spite of this reasonable agreement between our data and these predictions for the $\mathrm{T}$ dependence of $\delta_{\pi-0} \chi^{\prime \prime}(T)$, $\chi^{\prime \prime}$ displays a phase-dependence sharper than expected, for all frequencies investigated above the minigap (see fig. 12). This suggests that the contribution of the phasedependence of the squared matrix elements of the current operator still play a role even at rather large energy $\left(\hbar \omega \sim 3 E_{g}\right)$. Including an additional contribution due to the e-h symmetric transitions with a phase-dependence peaked at $\pi$ gives a better agreement (not shown) at the cost of introducing additional parameters. This argument is supported by numerical simulations, compare for
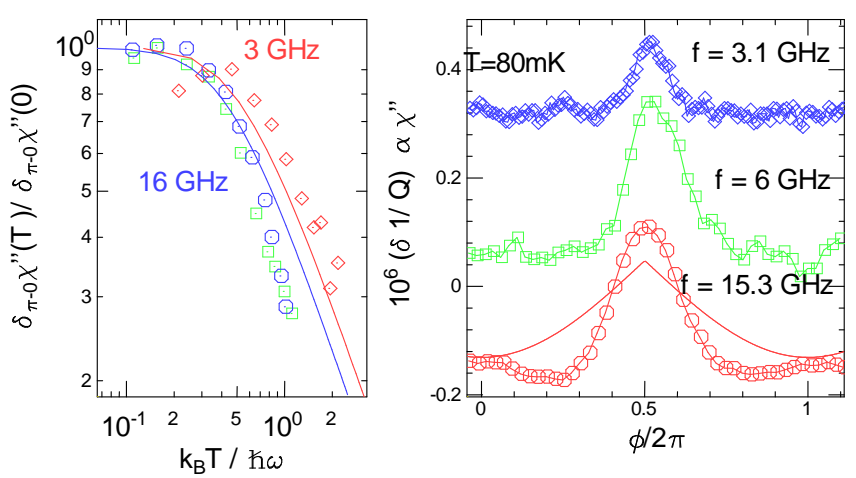

FIG. 12: Phase and temperature dependences of the dissipation at $T<E_{g} / k_{B}$. Left: Temperature dependence of $\delta_{\pi-0} \chi^{\prime \prime}$ at 16 (squares), 15.3 (circles) and $6 \mathrm{GHz}$ (diamonds, we note a saturation at temperatures corresponding to $0.25 \hbar \omega$. The plain lines correspond to equation 10 valid for $\hbar \omega \gg E_{g}$. Right: Phase dependence $\chi_{N D}(\varphi)$ measured for sample B at $80 \mathrm{mK}$ for different frequencies below and above the minigap corresponding to $5 \mathrm{GHz}$ in comparison with the phase-dependent minigap (plain line). The dissipation peak reflects closing of the minigap but the overall phase dependence has a shape which differs from the minigap, indicating a sizable contribution of the phase dependence of non-diagonal matrix elements.

instance $\chi "(15.3 \mathrm{GHz}, 80 \mathrm{mK})$ with fig.7 in [14]. Such a description requires requires a more quantitative analysis of the energy correlations of the $\left|J_{n m}\right|^{2}$ and is is left for future studies.

\section{CONCLUSION}

To summarize, we have followed the frequencydependent magnetic susceptibility of an NS ring from the adiabatic regime, where the coherent response is simply the phase-derivative of the current-phase relation, to the highly non-adiabatic regime where dynamical effects lead to dissipation. When the frequency is close to the inelastic scattering rate, the relaxation of populations is the dominant dissipative process. This yields a phasedependent dissipative response proportional to the inelastic time which is maximum around $\pi / 2$. When the frequency is high enough compared to the relaxation rate of the populations $1 / \tau_{i n}$, dissipation is dominated by transitions across the minigap. Dissipation is then related to the dynamics of coherence (non-diagonal terms of the density matrix) but is independent of their relaxation time in the limit of a continuous spectrum. Due to the closing of the minigap, this process leads to an absorption peak at $\varphi=\pi$. In both cases dissipation measured by the real part of the admittance reaches values which are paradoxically much greater than the normal state conductance. We attribute this increase to enhanced transitions between electron-hole symmetric states. The phase 


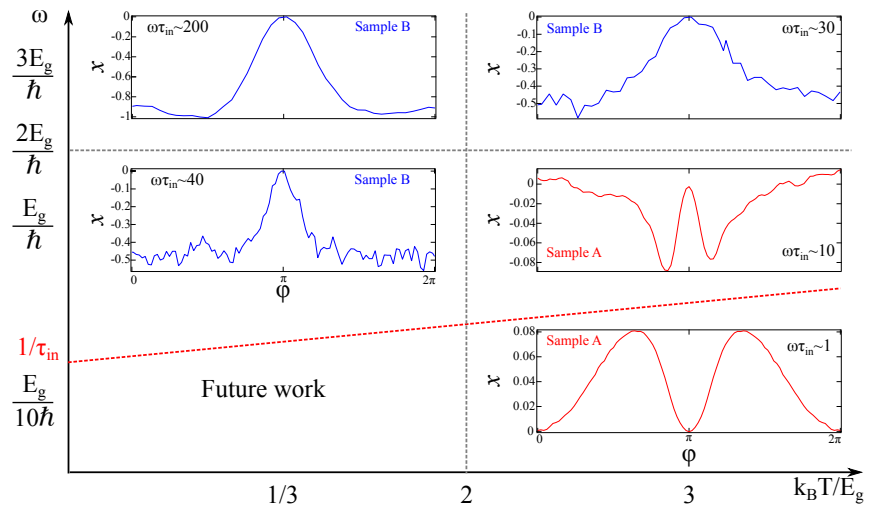

FIG. 13: Summary of the characteristic phase dependence of $\chi^{\prime \prime}$ observed depending on the different energy scales in the system. The normalized susceptibility $x=\frac{\chi^{\prime \prime}(\varphi)-\chi^{\prime \prime}(\pi)}{2 E_{g} G_{N} / \hbar}$ is plotted where $E_{g} / k_{B}=210 \mathrm{mK}$ and $G_{N}=1 \mathrm{~S}$ have been used for sample $\mathrm{A}$ and $2 E_{g} G_{N} / \hbar=$ $\delta_{\pi-0} \chi "(16 \mathrm{GHz}, 80 \mathrm{mK})$ for sample B.

dependences of these two contributions contain different physics summarized in fig.13. Whereas the diagonal contribution is proportional to the square of the single level current, the non-diagonal contribution is sensitive to the phase-dependent minigap at low temperature. At high temperature compared to the minigap the phase dependence $\chi^{\prime \prime}(\varphi)$ is determined by the matrix elements of the current operator between different Andreev states. These experiments constitute therefore a powerful tool for the investigation of the Andreev spectrum of a SNS junction. We propose to apply this method to reveal breaking of spin degeneracy, protected crossings and topological superconductivity in Josephson junctions with large spinorbit interactions [52-56].

We acknowledge A. Kasumov and F. Fortuna for help with the FIB and R. Danneau for suggesting the use of a conducting espacer. We are also grateful to M. Aprili, F. Chiodi, R. Deblock, M. Feigelman, T.T. Heikkilä, K. Tikhonov and P. Virtanen for fruitful discussions.

\section{APPENDIX A}

\section{Overview of theoretical predictions.}

Following previous work on the dynamics of persistent currents in normal mesoscopic Aharonov-Bohm rings [18, 57 we developed a Kubo formula description of the linear response of an NS ring to a time dependent AharonovBohm flux. In this approach, $\chi$ splits naturally into three parts, $\chi=\chi_{J}+\chi_{D}+\chi_{N D}$, as written in eq. 6 and detailed in the following.

\section{a) Josephson susceptibility $\chi_{J}$ : adiabatic response}

In the adiabatic regime $(\omega \rightarrow 0)$ the susceptibility stems from the phase dependence of the ABS and does not depend on frequency. It is purely non-dissipative and is the derivative of the Josephson supercurrent. At temperature high enough to suppress its higher harmonics $\left(k_{B} T \gtrsim E_{g}\right)$ the current-phase relation (CPR) of a long diffusive SNS junction is purely sinusoidal $[9,49]$ and the Josephson susceptibility reads

$$
\chi_{J}=-\frac{2 \pi}{\Phi_{0}} \frac{\partial I_{J}}{\partial \varphi}=-\frac{2 \pi I_{c}}{\Phi_{0}} \cos (\varphi)
$$

where $I_{c}(T)$ at high temperature [40] can be approximated by $I_{c}(T)=I_{J}(0) \exp \left(-k_{B} T / 3.6 E_{T h}\right)$ where the zero temperature Josephson current reads: $I_{J}(0)=$ $g_{N} 10.8 E_{T h} / \Phi_{0}$, with $g_{N}=G_{N} h / 2 e^{2}$ is the dimensionless conductance.

In the following we discuss the extra contributions to the susceptibility when increasing frequency. Two characteristic frequency scales emerge the inverse relaxation time of the populations $\tau_{i n}^{-1}$ and the inverse diffusion time $\tau_{D}^{-1}$, proportional to the minigap $E_{g}$.

b) Diagonal susceptibility $\chi_{D}$ : relaxation of $A B S$ populations

We discuss here $\chi_{D}$ called diagonal contribution because it only involves the diagonal elements of the current operator. It is the non-adiabatic contribution due to the thermal relaxation of the populations $f_{n}$ of the Andreev levels with the characteristic inelastic time $\tau_{\text {in }}$. It is related to the phase-sensitivity of the spectrum and is therefore correlated to the existence of non-dissipative currents at equilibrium [19]. Note that this contribution is specific to systems with a phase-dependent spectrum [57] and is ignored in most derivations of the Kubo formula. To first approximation it is proportional to the sum over an energy range $k_{B} T$ around the Fermi energy of the square of the single level current $i_{n}^{2}$. It can be recast as the product of a phase-independent term by a frequency-independent term

$$
\chi_{D}(\omega, \varphi, T)=A(\omega, T) F(\varphi, T)
$$

with $A(\omega)=-\frac{i \omega}{\tau_{i n}^{-1}-i \omega}$ and $F(\varphi, T)=-\sum_{n} i_{n}^{2} \frac{\partial f_{n}}{\partial \epsilon_{n}}$. At high temperature compared to $E_{T h}, F(\varphi, T)$ decays as $1 / T$ due to the energy derivative of the Fermi distribution. $F(\varphi, T)$ was first introduced by Lempitsky [41] to describe the $\mathrm{I}(\mathrm{V})$ characteristics of SNS junctions and was calculated numerically using time dependent Usadel equations by Virtanen et al.[13].

At high temperature compared to $E_{T h}, F(\varphi)$ can be 
approximated by the following analytical form:

$$
\begin{aligned}
& F_{U}(\varphi) \propto \frac{g_{N}}{k_{B} T} \frac{E_{g}^{2}}{\Phi_{0}^{2}} \\
& {\left[(-\pi+(\pi+\varphi)[2 \pi]) \sin (\varphi)-|\sin (\varphi)| \sin ^{2}(\varphi / 2) / \pi\right] .}
\end{aligned}
$$

It is dominated by its second harmonics with in addition a sharp linear singularity at odd multiples of $\pi$. This is due to the dominant contribution of Andreev levels close to the minigap whose flux dependence is singular as in a highly transmitting superconducting single channel point contact [58]. It is zero at $\varphi=\pi$ and $\varphi=0$, since single level currents go to zero at those phases. It was recently shown that $\chi_{D}$ is very sensitive to the presence of unavoided levels crossings in systems with spin-orbit interaction, and can be therefore useful to reveal topological superconductivity [55].

c) Non-diagonal susceptibility $\chi_{N D}$ : microwave induced transitions

We now consider $\chi_{N D}$, the contribution that describes microwave induced transitions within the Andreev spectrum. In the limit of a dense spectrum with level spacing $\delta_{E} \ll \hbar / \tau_{N D}, \chi_{N D}^{\prime \prime}(\varphi)$ is independent of $\tau_{N D}$ and reads:

$$
\chi_{N D}^{\prime \prime}=-\omega \sum_{n, m \neq n}\left|J_{n m}\right|^{2} \frac{f_{n}-f_{m}}{\epsilon_{n}-\epsilon_{m}} \delta\left(\epsilon_{n}-\epsilon_{m}-\hbar \omega\right)
$$

that becomes in the continuous spectrum limit:

$$
\begin{aligned}
& \chi_{N D}^{\prime \prime}=-\omega \iint d \epsilon d \epsilon^{\prime} n(\epsilon, \varphi) n\left(\epsilon^{\prime}, \varphi\right) \\
& {\left[\left|J\left(\varphi, \epsilon, \epsilon^{\prime}\right)\right|^{2} \frac{f(\epsilon)-f\left(\epsilon^{\prime}\right)}{\epsilon-\epsilon^{\prime}} \delta\left(\epsilon-\epsilon^{\prime}-\hbar \omega\right)\right]}
\end{aligned}
$$

where we have introduced the phase-dependent density of states $n(\epsilon, \varphi)=n_{0}\left[\theta\left(\epsilon-E_{g}(\varphi)\right)+\theta\left(-\epsilon-E_{g}(\varphi)\right)\right]$ with $\theta$ the Heaviside distribution. $J\left(\varphi, \epsilon, \epsilon^{\prime}\right)$ is the equivalent of $J_{n m}(\varphi)$ in the continuous limit.

We can distinguish two mechanisms involved in the phase dependence of $\chi_{N D}$. One is related to the phase dependence of the density of states whereas the second one is related to the phase dependence of the non-diagonal elements of the current operator $J_{n m}(\varphi)$. When frequency is smaller or of the order of the minigap it is important to consider the phase dependence of the matrix elements $J_{n m}(\varphi)$ which is determined by selection rules. In the case of a long and diffusive junction numerical simulations indicates that matrix elements between electron-hole symmetric transitions are greater than for non-symmetric ones [14]; these selection rules are even more stringent in ballistic systems or in the presence of spin-orbit interaction since no transitions are possible between Andreev states of opposite spin polarization $[53,55]$.

\section{Low temperature}

Let's consider now the low temperature regime such that $E_{g}(\varphi) \gg k_{B} T$. No transitions are possible for $\hbar \omega<E_{g}(\varphi)$ and dissipation drops in the corresponding range of phase. As a result in the limit of zero temperature and zero frequency dissipation is expected to be a delta peak at $\pi$. This peak is found in the resolution of time-dependent Usadel equations $[13,16]$ $G(\pi)=Y^{\prime}(\pi)=\chi^{\prime \prime}(\pi) / \omega$ is found increase at low temperature approximately like $G_{N}\left(1+A E_{g} / k_{B} T\right)$, with $A$ a coefficient of the order of unity, and to saturate at temperatures such that $k_{B} T \lesssim \hbar \omega$. Numerical simulations in [14] show that the main contribution to this dissipation peak at $\pi$ comes from $J_{n,-n}$ non-diagonal matrix elements of the current operator connecting electron-hole symmetric states whose energy is of the order of the minigap. We attribute the increase of $G(\pi, T)$ at low temperature and low frequency to these enhanced transitions.

In contrast at high frequency, when $\hbar \omega \gg E_{g}(0) \gg$ $k_{B} T, \chi_{N D}^{\prime \prime}$ is dominated by the high energy contribution of $J_{n m}$ which are independent of phase and the phasedependent absorption is exclusively determined by the density of states. It is therefore found to vary like:

$$
\chi_{N D}^{\prime \prime}(\varphi, \omega)=\left(\omega-\frac{2 E_{g}(\varphi)}{\hbar}\right) G_{N}
$$

where we have used that $\left\langle\left|J_{n m}\right|>^{2} n^{2}\left(E_{F}\right)\right.$ is the classical Drude conductance $G_{N}=\left(2 e^{2} / h\right) g_{N}$ of the normal wire. (The average $\left\langle\left|J_{n m}\right|>\right.$ being taken on an energy scale much larger than the superconducting gap but smaller than $\hbar / \tau_{e}$ where $\tau_{e}$ is the elastic scattering time). We find that at low temperature and high frequency compared to $E_{g}$ one can reveal the minigap since $\delta_{\varphi-0} \chi_{N D}^{\prime \prime}(\varphi, \omega)=-2 G_{N} \frac{E_{g}(\varphi)}{\hbar}$. It is independent of $\omega$ and $T$.

\section{High temperature}

In the limit of temperatures higher than the minigap, the phase dependence of the density of states can be neglected and the phase dependence $\chi_{N D}^{\prime \prime}(\varphi)$ is then dominated by the contributions $\sum\left|J_{n m}(\varphi)\right|^{2} \delta\left(\epsilon_{n}-\right.$ $\left.\epsilon_{m}-\hbar \omega\right)$. One can then write: $\delta_{\varphi} \int d \omega \chi_{N D}^{\prime \prime}(\varphi, \omega) / \omega=$ $\delta_{\varphi} \sum_{n, m \neq n}\left|J_{n m}(\varphi)\right|^{2}=\delta_{\varphi}\left\{\operatorname{Tr}\left(J^{2}\right)-\sum_{n}\left|J_{n n}(\varphi)\right|^{2}\right\}$

Moreover, since $\operatorname{Tr}\left(J^{2}\right)$ is phase-independent, the phase dependences of $\sum_{n \neq m}\left|J_{n n}\right|^{2}$ and $\sum_{n}\left|J_{n n}\right|^{2}$ are exactly opposite from one another. Numerical simulations [14] and time-dependent Usadel equations [16] indicate that for $\hbar \omega \leq E_{g} \leq k_{B} T$ the phase dependence of $\chi_{N D}^{\prime \prime}$ is independent of frequency and varies like $\delta_{\varphi-0} \chi_{N D}^{\prime \prime}=\left(-\hbar \omega / E_{g}\right) F_{U}(\varphi)$. 


\section{d) Relations between dissipative and non-dissipative phase-dependent contributions}

In the following we show that simple relations hold between the dissipative and non-dissipative contributions of $\delta_{\pi-0} \chi$ when $\omega \gg \tau_{i n}$. They can be obtained from eq.15 for $\chi_{N D}^{\prime \prime}$ and the following expression of $\chi^{\prime}$ :

$$
\begin{aligned}
& \delta_{\varphi-0} \chi^{\prime}=-\delta_{\varphi-0} \iint d \epsilon d \epsilon^{\prime} n(\epsilon, \varphi) n\left(\epsilon^{\prime}, \varphi\right) \\
& {\left[\left|J\left(\varphi, \epsilon, \epsilon^{\prime}\right)\right|^{2} \frac{f(\epsilon)-f\left(\epsilon^{\prime}\right)}{\epsilon-\epsilon^{\prime}}\right]}
\end{aligned}
$$

The phase-dependent

contribution $\delta_{\varphi-0}\left|J\left(\varphi, \epsilon, \epsilon^{\prime}\right)\right|^{2} n(\epsilon, \varphi) n\left(\epsilon^{\prime}, \varphi\right)$ is approximated by $g^{+}\left(\epsilon^{+} / \epsilon_{c}^{+}\right) \times g^{-}\left(\epsilon^{-} / \epsilon_{c}^{-}\right)$where $g^{+}$and $g^{-}$are functions of $\epsilon^{+}=\left(\epsilon+\epsilon^{\prime}\right) / 2$ and $\epsilon^{-}=\left(\epsilon-\epsilon^{\prime}\right) / 2$. The correlation functions $g^{+}$and $g^{-}$are expected to oscillate and decay on energy scales $\epsilon_{c}^{+}$and $\epsilon_{c}^{-}$of the order of $E_{g}$. Moreover in the limit where $k_{B} T \gg \epsilon^{-}$one can approximate in both integrals the function $\frac{f(\epsilon)-f\left(\epsilon^{\prime}\right)}{\epsilon^{-}}$by a square function centered on $\epsilon^{+}$of width $4 k_{B} T$ which yields for $\hbar \omega \ll E_{g} \ll k_{B} T:$

$$
\begin{aligned}
& \delta_{\varphi-0} \chi^{\prime \prime}(\omega) \simeq \omega G_{N} \int_{0}^{2 k_{B} T} g^{+}\left(\epsilon^{+} / \epsilon_{c}^{+}\right) d \epsilon^{+} \\
& \delta_{\varphi-0} \chi^{\prime} \simeq G_{N} / \hbar \int_{0}^{\infty} g^{-}\left(\epsilon^{-} / \epsilon_{c}^{-}\right) d \epsilon^{-} \int_{0}^{2 k_{B} T} g^{+}\left(\epsilon^{+} / \epsilon_{c}^{+}\right) d \epsilon^{+}
\end{aligned}
$$

From these expressions it is possible to deduce a simple general relation between $\delta_{\varphi-0} \chi_{N D}^{\prime \prime}$ and $\delta_{\varphi-0} \chi^{\prime}=\delta_{\varphi-0} \chi_{J}$ valid for $\omega \tau_{i n} \gg 1$.

$$
\delta_{\varphi-0} \chi_{N D}^{\prime \prime}=\left(\hbar \omega / \epsilon_{c}^{-}\right) \delta_{\varphi-0} \chi^{\prime} \simeq\left(\hbar \omega / E_{g}\right) \delta_{\varphi-0} \chi^{\prime}(T, \varphi)
$$

In particular for $\varphi=\pi$ we can use that $\delta_{\pi-0} \chi_{D}^{\prime}=0$ and $\delta_{\pi-0} \chi^{\prime}=\delta_{\pi-0} \chi_{J} \propto \exp \left(-k_{B} T / 1.16 E_{g}\right) . \quad \delta_{\pi-0} \chi_{N D}^{\prime \prime}$ is therefore expected to decrease exponentially with $\mathrm{T}$ like the Josephson current. For other values of $\varphi$, assuming that $\epsilon_{c}^{-} \simeq E_{g}$ this result yields the expected phase dependence of $\delta \chi_{N D}^{\prime \prime}(\varphi)=-\left(\omega / E_{g}\right) \delta \chi_{D}^{\prime}(\varphi)=-\left(\omega / E_{g}\right) F_{U}(\varphi)$ in this range of parameters, $\hbar \omega \ll E_{g} \ll k_{B} T$, leading to a maximum of $\delta \chi_{N D}^{\prime \prime}(\varphi)$ of the order of:

$$
\delta \chi_{N D}^{\prime \prime}=\left(I_{J}(0) / \Phi_{0}\right)\left(\hbar \omega / k_{B} T\right)=G_{N} \omega E_{g} /\left(k_{B} T\right)
$$

i.e. much higher than $\delta_{\pi-0} \chi_{N D}^{\prime \prime}$.

These relations between $\delta_{\varphi-0} \chi_{N D}^{\prime \prime}$ and $\delta_{\varphi-0} \chi^{\prime}$ are useful for the interpretation of our experimental data in the range of parameters where screening effects distort the phase dependence of $\chi_{m}^{\prime \prime}$. They can be understood as the Kramers-Kronig relation between the dissipative and non-dissipative components of the phase-dependent susceptibility. Extrapolating this reasoning to lower temperature and higher frequency, we expect that the ratio $\delta_{\pi-0} \chi_{N D}^{\prime \prime}(\omega) / \delta_{\pi-0} \chi^{\prime}$ will increase at low temperature (in agreement with the experimental results on sample B, see fig.5) but its calculation is complicated by logarithmic singularities in $\chi^{\prime}$, and is beyond the scope of this paper.

\section{APPENDIX B: DETERMINATION OF THE ABSOLUTE VALUE OF $\chi^{\prime \prime}(\pi)$}

We focus on the range of parameters such that $L_{l} \chi^{\prime \prime}(\varphi)$ can be neglected in eq.3 leading to simplified relations between $\chi_{m}$ and $\chi$ :

$$
\begin{aligned}
& \chi_{m}^{\prime}(\varphi)=\chi^{\prime}(\varphi) /\left(1-L_{l} \chi^{\prime}(\varphi)\right) \\
& \chi_{m}^{\prime \prime}(\varphi)=\chi^{\prime \prime}(\varphi) /\left(1-L_{l} \chi^{\prime}(\varphi)\right)^{2}
\end{aligned}
$$

From these relations one can easily deduce the ratio $R_{m}=\delta_{\pi-0} \chi_{m}^{\prime \prime} / \delta_{\pi-0} \chi_{m}^{\prime}$. This quantity is interesting since it does not depend on the coupling coefficient $L_{c}^{2}$ between the measured and intrinsic susceptibility which is not easy to estimate accurately. It reads:

$$
R_{m}=\frac{2 \chi^{\prime \prime}(\pi, T) L_{l}}{1-\beta^{2}}+\frac{\delta_{\pi-0} \chi^{\prime \prime}}{\delta_{\pi-0} \chi^{\prime}} \frac{1-\beta}{1+\beta}
$$

The first contribution to $R_{m}$ is proportional to $L_{l}$ and thus is due to screening. The denominator contains a term in $\beta^{2}$ which is negligible in the range of temperature we have investigated $(\beta \leq 0.4)$. The second contribution is proportional to the intrinsic ratio between $\delta_{\pi-0} \chi^{\prime \prime}$ and $\delta_{\pi-0} \chi^{\prime}$. Theoretical predictions (see Appendix A) indicate that it is reasonable to consider that $\chi^{\prime \prime}(0)=\chi^{\prime \prime}(\pi)$ when $T>2 E_{g}$, we therefore assume $\delta_{\pi-0} \chi^{\prime \prime}=0$. Under this assumption and within first order in $\beta$ equation 22 yields:

$$
R_{m}=2 \chi^{\prime \prime}(\pi, T) L_{l}
$$

Therefore we have shown how to deduce the value of $\chi^{\prime \prime}(\pi, T)=R_{m}(T) / 2 L_{l}$ shown in fig.10 from the measurement of $R_{m}(T)$ and the estimation of $L_{l}$.

\section{APPENDIX C: HIGH FREQUENCY DATA ON SAMPLE A, FOR WHICH SCREENING CORRECTIONS CANNOT BE QUANTITATIVELY DETERMINED}

For very high frequency (above $5 \mathrm{GHz}$ ) the screening corrections quadratic in $L_{l} \chi^{\prime \prime}$ in equations 2 and 3 cannot be neglected on sample $\mathrm{A}$ and give rise to an important intrication between the phase dependence of $\chi^{\prime}(\varphi)$ and $\chi^{\prime \prime}(\varphi)$ at low temperature and high frequency. This is illustrated below where $\chi_{m}^{\prime}$ exhibits a sharp split peak at $\pi$ as seen in fig.14. This intriguing phase dependence 

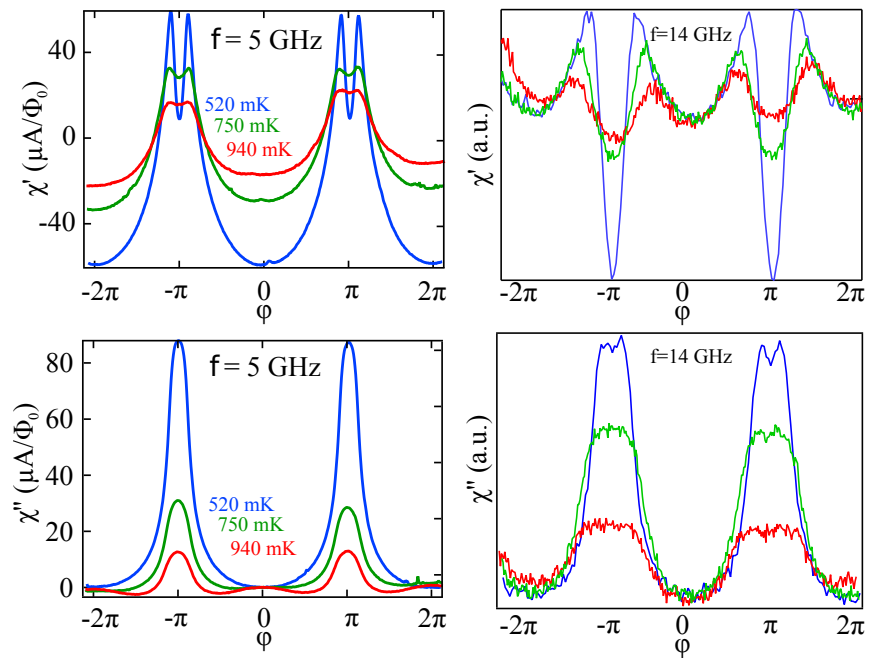

FIG. 14: Evolution of the phase dependence of $\chi_{m}^{\prime}$ (top) and $\chi "{ }_{m}$ (bottom) with temperature at $5 \mathrm{GHz}$ and $14 \mathrm{GHz}$ measured on sample A. Dips in $\chi_{m}^{\prime}$ at $\pi$ are attributed to screening effects. Data in this regime could not be corrected.
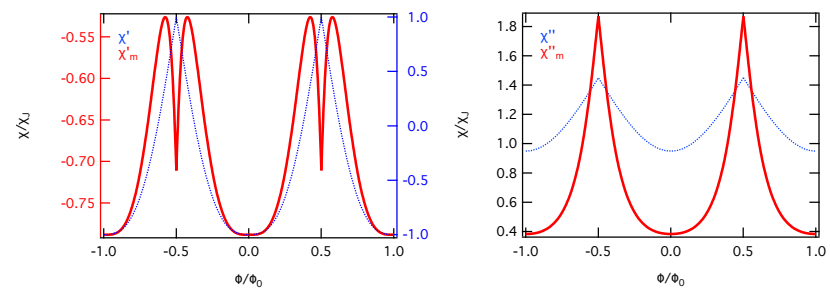

FIG. 15: Computed phase dependences of $\chi_{m}^{\prime}$ and $\chi_{m}^{\prime \prime}$ assuming $\chi^{\prime}=-\cos \varphi+\chi_{D}$ and $\chi^{\prime \prime}(\varphi)$ similar to $E_{g}(\varphi)$. One sees that $\chi_{m}^{\prime}$ exhibits a dip at $\pi$ and what $\chi_{m}^{\prime \prime}$ is much larger than $\chi^{\prime \prime}$ when mixing between $\chi^{\prime}$ and $\chi^{\prime \prime}$ contributions in $\chi_{m}^{\prime}$ and $\chi_{m}^{\prime \prime}$ (see Eqs.4 and 5) becomes important.

can be shown to be due to the negative term in expression 5 proportional to $-\chi^{\prime \prime}(\varphi)^{2}$ as illustrated in fig. 15 . Correlatively $\chi_{m}^{\prime \prime}(\varphi)$ exhibits a very sharp increase at $\pi$

[1] M. Büttiker, Y. Imry, and R. Landauer, Physics letters a 96, 365 (1983).

[2] L. Levy, G. Dolan, J. Dunsmuir, and H. Bouchiat, Physical review letters 64, 2074 (1990).

[3] V. Chandrasekhar, R. Webb, and M. Brady, Physical review Letters 67, 3578 (1991).

[4] D. Mailly, C. Chapelier, and A. Benoit, Physical Review Letters 70, 2020 (1993).

[5] J. Bardeen and J. Johnson, Physical Review B 5, 72 (1972).

[6] J. Waldram and J. Lumley, Revue de Physique Appliquée 10, 94 (1975).

[7] M. Büttiker and T. Klapwijk, Physical Review B 33, 5114 (1986).

[8] M. Della Rocca, M. Chauvin, B. Huard, H. Pothier, D. Esteve, and C. Urbina, Physical Review Letters 99,
127005 (2007)

[9] M. Fuechsle, J. Bentner, D. Ryndyk, M. Reinwald, W. Wegscheider, and C. Strunk, Physical Review Letters 102, 127001 (2009).

[10] R. Deblock, Y. Noat, B. Reulet, H. Bouchiat, and D. Mailly, Physical Review B 65, 075301 (2002).

[11] F. Chiodi, M. Ferrier, K. Tikhonov, P. Virtanen, T. Heikkilä, M. Feigelman, S. Guéron, and H. Bouchiat, Scientific reports 1, 3 (2011).

[12] B. Dassonneville, M. Ferrier, S. Guéron, and H. Bouchiat, Physical Review Letters 110, 217001 (2013).

[13] P. Virtanen, F. S. Bergeret, J. Cuevas, and T. Heikkilä, Physical Review B 83, 144514 (2011).

[14] M. Ferrier, B. Dassonneville, S. Guéron, and H. Bouchiat, Phys. Rev. B 88, 174505 (2013).

[15] Doru Sticlet and Jérome Cayssol Phys. Rev. B 90, 201303(R) (2014).

[16] K. S. Tikhonov and M. V. Feigel'man, Physical Review B 91, 054519 (2015).

[17] D. Fisher and P. Lee, Physical Review B 23, 6851 (1981).

[18] B. Reulet and H. Bouchiat, Physical Review B, 2259 (1994).

[19] M. Büttiker, Physica Scripta T14, 82 (1986).

[20] M Büttiker, Flux-Sensitive Effects in Normal Metal Loops. Annals of the New York Academy of Sciences, 480, 209 (1986).

[21] B. Reulet, Ph.D. thesis (1994).

[22] R. Deblock, Ph.D. thesis (2001).

[23] M. A. Despósito and A. Levy Yeyati, Phys. Rev. B, 64, $140511(2001)$

[24] A. Zazunov, V. S. Shumeiko, E. N. Bratus, J. Lantz, \& G. Wendin, Phys. Rev. Lett. 90, 087003 (2003).

[25] C. Janvier, L. Tosi, L. Bretheau, Ç. Ö. Girit, M. Stern, P. Bertet, P. Joyez, D. Vion, D. Esteve, M. F. Goffman, H. Pothier, C. Urbina, Science 349, 1199 (2015)

[26] J.-D. Pillet, C. H. L. Quay, P. Morfin, C. Bena, A. L. Yeyati, and P. Joyez, Nature Physics 6, 965 (2010).

[27] L. Bretheau, C. O. Girit, H. Pothier, D. Esteve, and C. Urbina, Nature 499, 312 (2013).

[28] L. Bretheau, C. Girit, C. Urbina, D. Esteve, and H. Pothier, Physical Review X 3, 041034 (2013).

[29] David J van Woerkom, Alex Proutski, Bernard van Heck, Daniël Bouman, Jukka I Väyrynen, Leonid I Glazman, Peter Krogstrup, Jesper Nygård, Leo P Kouwenhoven, and Attila Geresdi, Nature Physics 13, 876 (2017).

[30] T. Heikkilä, J. Särkkä, and F. Wilhelm, Physical Review B 66, 184513 (2002).

[31] F. Zhou, P. Charlat, B. Spivak, and B. Pannetier, Journal of Low Temperature Physics 110, 841 (1998).

[32] D. Ivanov, R. von Roten, and G. Blatter, Physical Review B 66, 052507 (2002).

[33] H. le Sueur, P. Joyez, H. Pothier, C. Urbina, and D. Esteve, Physical Review Letters 100, 1 (2008)

[34] F.Zhou and B. Spivak Pis'ma Zh.Eksp.Teor.Fiz. 65, 347 (1997).

[35] R. Rifkin and B. S. Deaver, Physical Review B 13, 3894 (1976)

[36] M. Ebel, C. Busch, U. Merkt, M. Grajcar, T. Plecenik, and E. Il'ichev, Physical Review B 71, 052506 (2005).

[37] A. G. P. Troeman, S. H. W. Van Der Ploeg, E. Il'Ichev, H. G. Meyer, A. A. Golubov, M. Y. Kupriyanov, and H. Hilgenkamp, Physical Review B 77, 024509 (2008).

[38] Russell E. Lake, Joonas Govenius, Roope Kokkoniemi, 
Kuan Yen Tan, Matti Partanen, Pauli Virtanen, Mikko Möttönen Adv. Electron. Mater. 3, 1600227 (2017).

[39] B. Dassonneville, Ph. D. Thesis, Université Paris Sud Paris XI (2014).

[40] F. K. Wilhelm, A. D. Zaikin, and G. Schön, Supercurrent in a mesoscopic proximity wire, J. Low Temp. Phys. 106, 305 (1997).

[41] S. Lempitskii, Sov. Phys.-JETP (Engl. Transl.) 58, 624 (1983).

[42] This $1 / \mathrm{T}$ behavior is notably different from the power law given in [12]. This is due to the underestimation of screening effects leading to erroneous values of $\tau_{\text {in }}$ at low temperature.

[43] Y. Blanter. electron-electron scattering rate in disorder mesoscopic systems. Phys. Rev. B 54, 12807, (1997).

[44] C. Texier and G. Montambaux, Physical Review B 72, 115327 (2005).

[45] M. Ferrier, A. C. H. Rowe, S. Guéron, H. Bouchiat, C. Texier, and G. Montambaux, Physical Review Letters 100, 1 (2008).

[46] T. Capron, C. Texier, G. Montambaux, D. Mailly, A. Wieck, and L. Saminadayar, Physical Review B 87, 041307 (2013).

[47] K. Lehnert, N. Argaman, H.-R. Blank, K. Wong, S. Allen, E. Hu, and H. Kroemer, Physical Review Let- ters 82, 1265 (1999).

[48] N. Argaman, Superlattices and microstructures 25 (1999).

[49] P. Dubos, H. Courtois, O. Buisson, and B. Pannetier, Physical Review Letters 87, 206801 (2001).

[50] H. Courtois, M. Meschke, J. Peltonen, and J. Pekola, Physical Review Letters 101, 067002 (2008).

[51] A. De Cecco, K. Le Calvez, B. Sacp, C. B. Winkelmann, and H. Courtois, Physical Review B 93, 180505(R) (2016).

[52] Jukka I. Väyrynen, Gianluca Rastelli, Wolfgang Belzig, and Leonid I. Glazman, Phys. Rev. B 92, 134508 (2015).

[53] B. van Heck, J. I. Väyrynen, and L. I. Glazman Phys. Rev. B 96, 075404 (2017).

[54] Olesia Dmytruk, Mircea Trif, Pascal Simon Phys. Rev. B 94, 115423 (2016).

[55] Murani A., Chepelianskii A., Guéron, S. and Bouchiat H., Phys. Rev. B 96, 165415, (2017).

[56] Mircea Trif, Olesia Dmytruk, Helene Bouchiat, Ramón Aguado, Pascal Simon, arxiv 1708.03096 (2017).

[57] N. Trivedi and D. Browne, Physical Review B 38 (1988).

[58] A. Martín-Rodero, A. Levy Yeyati, and F. García-Vidal, Physical review. B 53, R8891 (1996). 